\title{
Rotational variation of daughter species production rates in Comet 103P/Hartley: Implications for the progeny of daughter species and the degree of chemical heterogeneity
}

\author{
Adam J. McKay ${ }^{\mathrm{a}, *}$, Nancy J. Chanover ${ }^{\mathrm{a}}$, Michael A. DiSanti ${ }^{\mathrm{b}}$, Jeffrey P. Morgenthaler ${ }^{\mathrm{c}}$, Anita L. Cochran ${ }^{\mathrm{d}}$, \\ Walter M. Harris ${ }^{\mathrm{e}}$, Neil Dello Russo ${ }^{\mathrm{f}}$ \\ a Astronomy Department, New Mexico State University, 1320 Frenger Mall, Las Cruces, NM 88001, USA \\ ${ }^{\mathrm{b}}$ Goddard Space Flight Center, 8800 Greenbelt Rd., Greenbelt, MD 20771, USA \\ c Planetary Science Institute, 1700 E. Fort Lowell, Ste 106, Tucson, AZ 85719, USA \\ ${ }^{\mathrm{d}}$ Univerisity of Texas Austin/McDonald Observatory, 1 University Station, Austin, TX 78712, USA \\ e Department of Applied Science, University of California Davis, 1 Shields Ave., Davis, CA 95616, USA \\ f Johns Hopkins University Applied Physics Laboratory, 11100 Johns Hopkins Rd., Laurel, MD 20723, USA
}

\section{A R T I C L E I N F O}

\section{Article history:}

Received 4 July 2013

Revised 22 November 2013

Accepted 22 November 2013

Available online 23 December 2013

\section{Keywords:}

Comets

Comets, coma

Comets, composition

\begin{abstract}
A B S T R A C T
We present analysis of high spectral resolution optical spectra of Comet 103P/Hartley taken during its Fall 2010 apparition. These spectra include transitions belonging to $\mathrm{CN}, \mathrm{C}_{2}, \mathrm{CH}, \mathrm{NH}_{2}$, and OI. We measure production rates and mixing ratios from these spectra. We find evidence for large changes in production rates (factors of a few) over the course of a nucleus rotation, in agreement with other measurements. We also measure variability with rotational phase in the $\mathrm{CN} / \mathrm{H}_{2} \mathrm{O}$ and $\mathrm{C}_{2} / \mathrm{CN}$ ratios, which has not been previously reported for any comet. There may also be variability in the $\mathrm{NH}_{2} / \mathrm{H}_{2} \mathrm{O}$ ratio with rotational phase, but this trend is not as clear as for $\mathrm{CN} / \mathrm{H}_{2} \mathrm{O}$. We interpret the changing mixing ratios as due to $\mathrm{H}_{2} \mathrm{O}$ and $\mathrm{C}_{2}$ being released primarily from the icy grain halo, while the $\mathrm{CN}$ parent molecule comes directly from the nucleus. There is evidence that the $\mathrm{CH} / \mathrm{CN}$ ratio is higher pre-perihelion than post-perihelion. We conclude that the observed $\mathrm{CN}$ and $\mathrm{NH}_{2}$ abundances are consistent with $\mathrm{HCN}$ and $\mathrm{NH}_{3}$ being the dominant parent molecules for these species. The $\mathrm{C}_{2}$ and $\mathrm{CH}$ abundances are higher than those of candidate parent molecules $\left(\mathrm{C}_{2} \mathrm{H}_{2}\right.$ and $\mathrm{CH}_{4}$ respectively), so there must be another source for these molecules in 103P's coma. Carbonaceous dust grains could serve as this source.
\end{abstract}

(c) 2013 Elsevier Inc. All rights reserved.

\section{Introduction}

Comets have a primitive composition that has undergone very little alteration since their formation. Therefore studies of cometary composition play a key role in understanding the formation and evolution of the early Solar System. Cometary volatile composition is best studied using in situ measurements by spacecraft flybys or a sample return mission, but even in aggregate these studies can investigate only a tiny portion of the cometary population. This makes ground-based observations of cometary comae critical for characterizing the population as a whole and placing mission results in context.

In order to infer the chemical composition of cometary nuclei from observations of volatile species in the coma, an understanding

\footnotetext{
* Corresponding author.

E-mail addresses: amckay@nmsu.edu (A.J. McKay), nchanove@nmsu.edu (N.J. Chanover), Michael.A.Disanti@nasa.gov (M.A. DiSanti), jpmorgen@psi.edu (J.P. Morgenthaler), anita@barolo.as.utexas.edu (A.L. Cochran), wmharris@ucdavis.edu (W.M. Harris), neil.dello.russo@jhuapl.edu (N.D. Russo).
}

of the physics and chemistry operating in the coma is needed, since these processes work to alter the coma composition. A key process operating in cometary comae is photodissociation of molecules as they travel away from the nucleus. This means that atomic and molecular species present in cometary comae can be classified into two main groups based on their provenance. Molecules released directly from the nucleus (or directly from the icy grain halo surrounding the nucleus) are defined as parent species. Parent species are inherently present as ices or trapped gases in the cometary nucleus. Examples of parent species in cometary comae are $\mathrm{H}_{2} \mathrm{O}, \mathrm{HCN}$, and $\mathrm{C}_{2} \mathrm{H}_{6}$. Molecules or atoms that are released into the coma by photodissociation of a parent molecule are called daughter species. Daughter species are not inherently present in the nucleus, but are released into the coma only upon photodissociation of another molecule. Examples of daughter species include OI, CN, and $\mathrm{C}_{2}$.

Parent-daughter inter-relationships in comets remain poorly understood. For instance, it has been noted in some comets that there is not enough $\mathrm{HCN}$ and $\mathrm{C}_{2} \mathrm{H}_{2}$ to account for the observed abundances of $\mathrm{CN}$ and $\mathrm{C}_{2}$, respectively (Dello Russo et al., 2009; 
Kobayashi et al., 2010), though in other comets this is not the case. It is possible that the excess $\mathrm{CN}$ and $\mathrm{C}_{2}$ in certain comets comes from carbonaceous dust grains or HCN polymers. Linking observations of parent and daughter species is an important step in addressing the origin of daughter species, and is best done with near simultaneous observations in the NIR and/or radio portions of the electromagentic spectrum (for characterization of the parent species) and the optical (to characterize the daughter species). However, the sample size of comets for which NIR and/or radio observations have been made is small $(\sim 20)$ and is not yet statistically significant. The sample size of comets for which nearly coincident optical and NIR/ radio data have been obtained is even smaller.

The degree of chemical heterogeneity in cometary nuclei can also influence the observed composition of the coma and has important consequences for the formation and evolutionary history of comets. Potentially observable consequences of heterogeous chemical composition in the nucleus include rotational modulation (short term) and seasonal effects (longer term) on the gas production rate, and spatial asymmetries in the distribution of gaseous species in the inner coma. Previous observations have revealed compositional heterogeneities in Comets 9P/Tempel (Feaga et al., 2007) and C/2007 W1 Boattini (Villanueva et al., 2011).

Comet 103P/Hartley (hereafter 103P) is a Jupiter Family Comet (JFC) that was the target of the Deep Impact Extended Investigation (DIXI) mission, which performed a flyby of the nucleus on UT November 4, 2010. Due to the favorable apparition of 103P in Fall 2010 ( $\Delta \sim 0.12$ AU at closest approach to Earth) and the DIXI flyby, this comet was the subject of a coordinated ground-based observational campaign to support the DIXI flyby (Meech et al., 2011). This provided a unique opportunity to correlate observations of the nucleus made by DIXI with high quality ground-based observations of the coma. Together the DIXI and ground-based observations can increase our understanding of coma physics and chemistry.

We present analysis of high resolution optical spectroscopy of 103P obtained in Fall 2010. 103P was also observed throughout the apparition using the near infrared spectrometer NIRSPEC on Keck II (Dello Russo et al., 2011; Mumma et al., 2011; Kawakita et al., 2013). The optical data presented here and the NIRSPEC observations of $103 \mathrm{P}$ provide a much needed near simultaneous optical and NIR spectral data set for a comet. The paper is organized as follows: in Section 2 we describe our observations and reduction and analysis procedures. Section 3 presents our results. In Section 4 we discuss our results in the context of the parentdaughter relationship and degree of chemical heterogeneity for 103P. Section 5 presents a summary of our conclusions.

\section{Observations and data analysis}

\subsection{Observations}

We obtained spectra of 103P using the ARCES echelle spectrometer mounted on the Astrophysical Research Consortium 3.5-m telescope at Apache Point Observatory (APO) in Sunspot, New Mexico. ARCES provides a spectral resolution of $R \equiv \frac{\lambda}{\Delta \lambda}=31,500$ and a spectral range of $3500-10,000 \AA$ with no interorder gaps. This large, uninterrupted spectral range allows for simultaneous observations of many gaseous species present in cometary comae, such as $\mathrm{CN}$ and $\mathrm{C}_{2}$. More specifics for this instrument are discussed elsewhere (McKay et al., 2012, 2013).

The observation dates and geometries are described in Table 1. All nights were photometric, meaning absolute flux calibration of the spectra was possible. We centered the $3.2^{\prime \prime} \times 1.6^{\prime \prime}$ slit on the optocenter of the comet. We used an ephemeris generated from
JPL Horizons for non-sidereal tracking of the optocenter. For short time-scale tracking, the guiding software uses a boresight guiding technique, which utilizes optocenter flux that falls outside the slit to keep the slit on the optocenter. We observed a G2V star in order to remove the underlying solar continuum and Fraunhofer absorption lines. We obtained spectra of an AOV star to account for telluric features and spectra of a flux standard to establish absolute intensities of cometary emission lines. The calibration stars used for each observation date are given in Table 1. To ensure comparable airmass for the comet and AOV star observations, we alternated between observing the AOV star and the comet throughout the night. We obtained spectra of a quartz lamp for flat fielding and acquired spectra of a ThAr lamp for wavelength calibration.

\subsection{Data reduction}

Spectra were extracted and calibrated using IRAF scripts that perform bias subtraction, cosmic ray removal, flat fielding, and wavelength calibration. We divided each comet, G2V, and flux standard star spectrum by the AOV star spectrum to remove telluric features. We then converted the tellurically corrected comet spectrum flux to physical units using the tellurically corrected flux standard spectrum. We assumed an exponential extinction law and extinction coefficients for APO when flux calibrating the cometary spectra. We shifted the tellurically corrected solar analog spectrum in wavelength to match the comet spectrum. Then we scaled the solar analog spectrum to the flux calibrated comet spectrum and subtracted the solar analog spectrum to remove Fraunhofer lines.

Because of the small size of the ARCES slit, it is necessary to obtain an estimate of the slit losses to achieve an accurate flux calibration. We find the transmittance through the slit by performing aperture photometry on the slit viewer images. For a given exposure, the initial slew places the target off the slit, so the initial slitviewer image serves as a reference flux for the subsequent images. During the exposure the slit is superimposed on the star, therefore comparison of the stellar flux in these images to the unobstructed star image gives an estimate of the transmittance. An example plot of calculated transmittance versus time for the flux standard used for our November 4 observations is shown in Fig. 1. The mean transmittance is plotted as a solid line, which we adopt as the transmittance through the slit for a given observation. We adopt the standard deviation of the transmittance calculated from the ensemble of slitviewer images on a given night as the 1-sigma uncertainty in our transmittance estimation, which in Fig. 1 is plotted as dashed lines. The transmittance is typically between $70-95 \%$ and the typical standard deviation in the transmittance estimation is approximately $10 \%$.

Another possible issue related to the small ARCES slit is the effect of differential refraction on fluxes obtained at different wavelengths. This is potentially important for $\mathrm{CN}$, whose primary emission band is in the near UV ( $3870 \AA$ ) and therefore suffers from significant differential refraction compared to other species like $C_{2}$ and [O I], which reside in the 5000-6500 $\AA$ range. To evaluate the effect of differential refraction, we employ the computed differential refraction as a function of wavelength and airmass from Filippenko (1982), and interpolate to the relevant wavelengths and airmasses for our observations. We calculate the amount of differential refraction and use a Haser model profile convolved with a Gaussian point spread function to estimate the slit loss due to the differential refraction. For all our observations, the effect of differential refraction is $5 \%$ or less. This is smaller than other uncertainties (e.g. slit losses), so we consider the effect of differential refraction on our results neglibible. 
Table 1

Details of $103 \mathrm{P} /$ Hartley observations.

\begin{tabular}{|c|c|c|c|c|c|c|c|c|c|}
\hline Date (UT) & UT times (or range) & $r(\mathrm{AU})$ & $\dot{r}\left(\mathrm{~km} \mathrm{~s}^{-1}\right)$ & $\Delta(\mathrm{AU})$ & $\dot{\Delta}\left(\mathrm{km} \mathrm{s}^{-1}\right)$ & Proj. FOV (km) & $\mathrm{G} 2 \mathrm{~V}$ & $\mathrm{AOV}$ & Flux Cal \\
\hline $9 / 8 / 2010$ & $6: 09-11: 22$ & 1.26 & -12.3 & 0.34 & -13.6 & $790 \times 395$ & HD 218633 & HR 8837 & $\mathrm{BD}+284211$ \\
\hline $9 / 30 / 2010$ & $4: 57-10: 48$ & 1.13 & -8.2 & 0.19 & -9.9 & $442 \times 221$ & HD 222794 & HD 5071 & $\mathrm{BD}+284211$ \\
\hline $10 / 1 / 2010$ & $2: 39-11: 09$ & 1.12 & -7.9 & 0.18 & -9.7 & $418 \times 209$ & HD 222794 & HD 5071 & $\mathrm{BD}+284211$ \\
\hline $11 / 04 / 2010$ & $7: 50-10: 13$ & 1.06 & 2.1 & 0.15 & 6.9 & $348 \times 174$ & HD 59711 & HD 56525 & Hilt 600 \\
\hline $11 / 26 / 2010$ & 9:39 & 1.13 & 8.3 & 0.26 & 8.8 & $604 \times 302$ & HD 59711 & HD 56525 & Hilt 600 \\
\hline
\end{tabular}

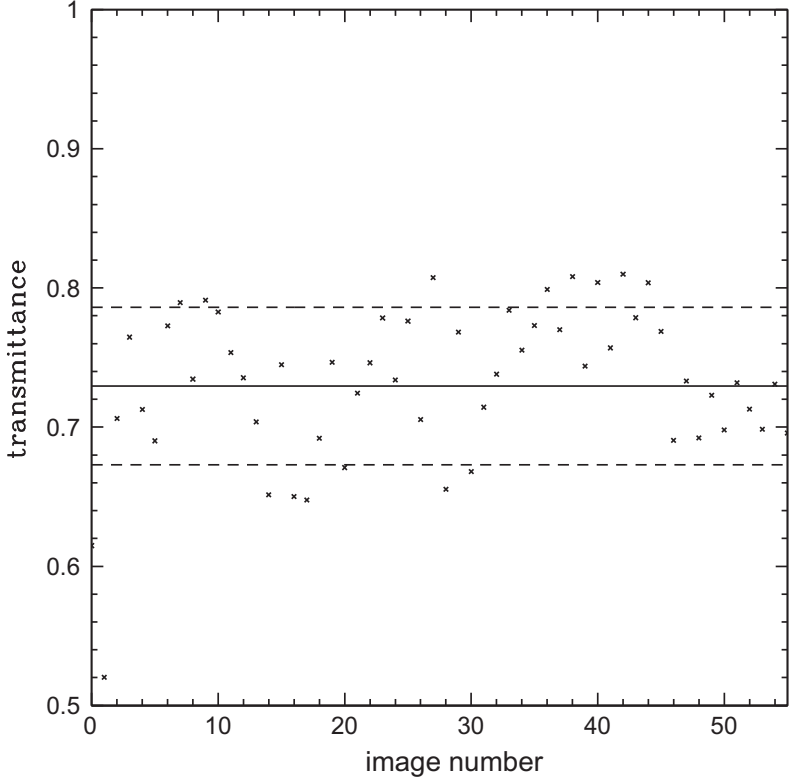

Fig. 1. Plot of transmittance versus image number for a series of slit viewer images of the flux standard star Hilt 600 on November 4, 2010. The solid line is the mean value of the transmittance, which is the value we adopt as the transmittance for a given observation. The dashed lines enclose the range of transmittances corresponding to the standard deviation of the data, which we adopt as the 1-sigma uncertainty in our slit loss estimation.

\subsection{Analysis of [O I] emission}

Among the emission features present in the ARCES bandpass is the [O I]6300 line. The [O I]6300 line is also present as a telluric emission feature, and so a combination of high spectral resolution and large geocentric velocity (and therefore large Doppler shift) is needed to resolve the cometary line from the telluric feature. For the observations reported here, only on November 4 are the telluric and cometary features not sufficiently separated. However, for bright comets like 103P the $6300 \AA$ line is much stronger than the telluric feature, so we can use the measured $6300 \AA$ line flux to estimate the $\mathrm{H}_{2} \mathrm{O}$ production rate, with the caveat that there is likely a small $(\sim 10 \%)$ correction needed to account for telluric contamination of the line flux. For the observations where the telluric and cometary [O I] emission were sufficiently separated, we deblended the lines using the method described in McKay et al. (2012) and McKay et al. (2013).

\subsection{Spectral fitting model for molecular species}

The transitions of interest in this work are electronic fluorescence transitions (except for [O I]6300, which is prompt emission). For molecules, there is ro-vibrational structure superimposed on the electronic transitions, which results in an intricate band structure for the emission features. These bands can include hundreds to thousands of lines spread out over spectral regions with widths

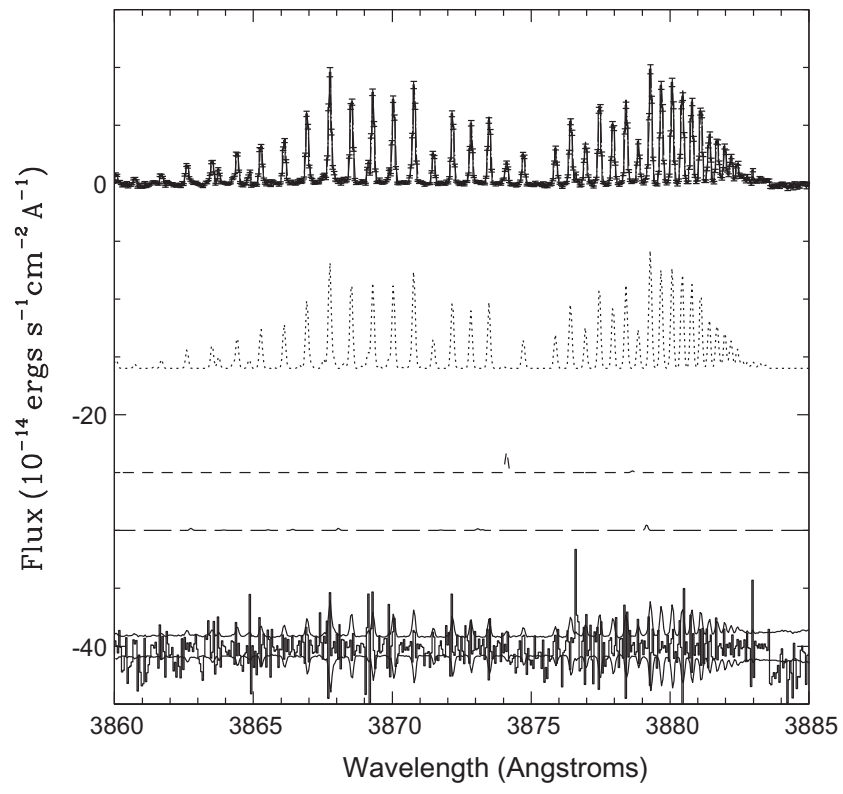

Fig. 2. Fit to the $3860-3885 \AA$ wavelength range for a spectrum taken on UT November 4 . The histogram represents the data, while the overplotted curve is the best model fit to the data. Below the data and fit are the individual fits for each species present: $\mathrm{CN}$ (dotted), $\mathrm{CH}$ (short dash), and unidentified lines (long dash), which are offset for clarity. At the bottom we plot the residuals of the model fit (multiplied by a factor of 10 for clarity) and a 1-sigma error envelope.

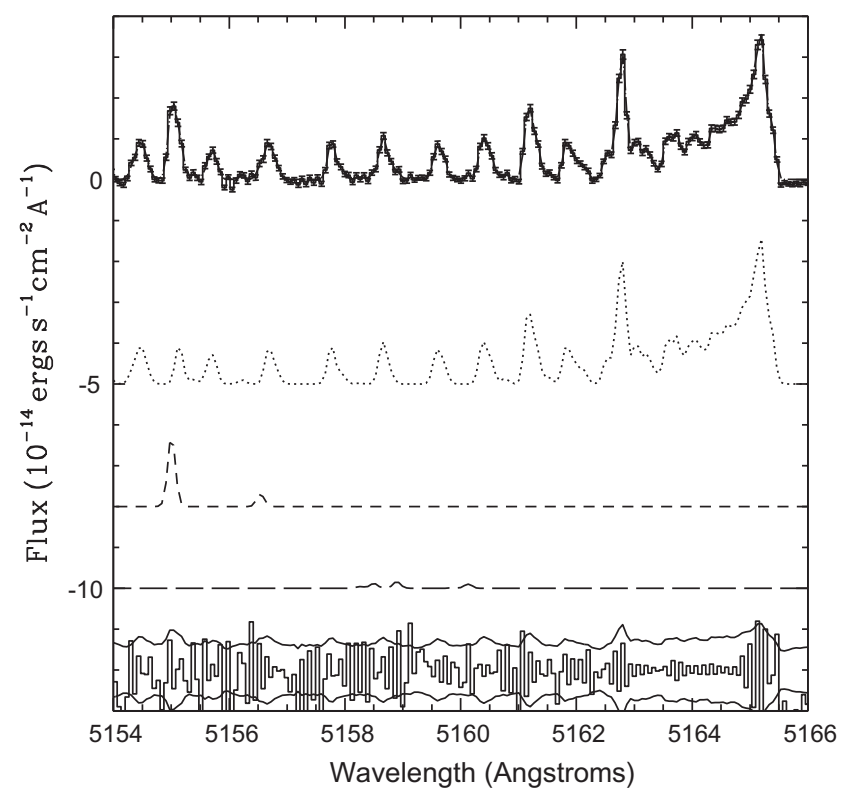

Fig. 3. Same as Fig. 2 except for the 5154-5166 A wavelength range. Here the individual species present are $\mathrm{C}_{2}$ (dotted), $\mathrm{NH}_{2}$ (short dash), and unidentified lines (long dash). 
of hundreds to thousands of Angstroms. This makes analyzing high spectral resolution observations of molecular daughter species in the optical in a consistent manner challenging.

For this purpose we developed a spectral fitting program specifically designed for ARCES spectra. The program references the line atlas for Comet 122P/De Vico compiled by Cochran and Cochran (2002) to find the rest wavelengths for the transitions of interest. Since the Cochran and Cochran (2002) line atlas has a higher spectral resolution than the ARCES data, many features listed as individual lines in the Cochran and Cochran (2002) line atlas blend together at the ARCES resolution and manifest themselves as a single line. We account for this blending when generating the final line list that the program uses to fit the line profiles. The rest wavelengths generated by this procedure are then shifted according to the geocentric velocity of the comet at the time of observation. At this point the number of spectral lines and the central wavelength for each spectral line match those in the ARCES spectra. Once this line list is compiled, we fit each spectral feature in the list to a Gaussian profile (i.e. the ISF, see Section 2.3). However, there are some cases where two or more lines can partially blend, resulting in a multi-peaked profile that is the sum of two or more Gaussians. We identify the lines for which this is an issue and fit the entire line profile to a sum of the appropriate number of Gaussians. We add all the line profile fits together to create an empirical fit to the spectrum and then integrate over this model fit to obtain a flux for the band of interest.

The spectral fitting is necessary because there is mixing between different species throughout the observed spectrum. For example, the wavelength range covered by the $C_{2} \Delta v=0$ band also contains $\mathrm{NH}_{2}$ emission. So integrating the data over this spectral range would include both $\mathrm{C}_{2}$ and $\mathrm{NH}_{2}$ lines, which results in an overestimation of the $C_{2}$ flux. Since we do not currently have a detailed fluorescence model for the species of interest, the model has no constraints on relative strengths of lines. Therefore a somewhat arbitrary hierarchy is used when determining line identifications for heavily blended lines with emission from multiple species. We performed tests with several hierarchies to evaluate the systematic error introduced. All cases produced small differences in the measured fluxes $(<7 \%)$. Example fits for the 3860$3885 \AA$ (dominated by the $\mathrm{CN} \Delta v=0$ band) and the 5154$5166 \AA$ ( $C_{2} \Delta v=0$ emission, but also $\mathrm{NH}_{2}$ and a couple unidentified lines) spectral regions are shown in Figs. 2 and 3, respectively.

\subsection{Conversion of observed flux to production rate}

The flux derived above is representative of the number of molecules present in the volume covered by our slit. In order to convert these fluxes into production rates, we need to extrapolate to the flux that would be measured if the entire coma was observed. This extrapolation is done by calculating an aperture correction. The aperture correction is the ratio of the total number of molecules in the entire coma (i.e. the total number of molecules contained in a FOV encompassing the entire coma) to the total number of molecules present in the slit FOV. To determine the aperture corrections for our observations, we employed algorithms based on those used in Morgenthaler et al. (2001), Morgenthaler et al. (2007), and McKay et al. (2012), which can be summarized as follows. We calculate the number density for the species of interest as a function of nucleocentric distance using the computationally simple Haser Model (Haser, 1957). The Haser Model gives the gas number density as a function of nucleocentric distance, given by

$n(r)=\frac{Q}{4 \pi r^{2} v} \frac{\beta_{p}}{\beta_{d}-\beta_{p}}\left(e^{-\beta_{d} r}-e^{-\beta_{p} r}\right)$ for a daughter species. Here $n$ is the number density, $r$ is the nucleocentric distance, $Q$ is the production rate, $v$ is the coma expansion velocity, and $\beta_{p}$ and $\beta_{d}$ are the inverse photodissociation scale lengths for the parent and daughter species, respectively. The photodissociative scale length is defined as

$\beta \equiv \frac{1}{v \tau}$

where $\tau$ is the photodissociation lifetime. The Haser Model is unphysical in that it assumes a spherically symmetric expanding gas cloud, and does not account for the isotropic distribution of ejection velocities for daughter species. Therefore we modify the Haser scale lengths following the prescriptions of Combi et al. (2004) to emulate the more physical vectorial model (Festou, 1981), which accounts for isotropic ejection of daughter species following dissociation of the parent molecule. Tseng et al. (2007) measured the expansion velocity of the gas coma to be $0.96 \mathrm{~km} \mathrm{~s}^{-1}$ at $1 \mathrm{AU}$; they also found that it scales as $r^{-0.44}$. Using the Tseng et al. (2007) relation, we derive expansion velocities of $0.87-0.94 \mathrm{~km} \mathrm{~s}^{-1}$ for the coma of 103P at the epochs of our observations. It is important to note that because of our small projected slit size, a large fraction of the gas may not be accelerated to the terminal value calculated using the Tseng et al. (2007) relation. However, as we assume the same expansion velocity for all species, this will only affect absolute production rates and not mixing ratios. Therefore our production rates may be biased by the assumption of constant expansion velocity, but the mixing ratios are not. The physical parameters we employ for each molecule are given in Table 2. The relevant parameters are the parent photodissociative lifetime $\tau_{p}$, the daughter photodissociative lifetime $\tau_{d}$, and the ejection velocity of the daughter after photodissociation of the parent $v_{e j}$. All scale lengths and excess velocities are adopted from Huebner et al. (1992). The lifetimes are given for a heliocentric distance of $1 \mathrm{AU}$.

Once the spatial distribution is calculated, we use a numerical integrator to convert the spatial density function to a column density along the line of sight for each point in the field of view (FOV). We use a similar integration technique to find the total number of molecules in the FOV. We changed the FOV from a circular aperture to a rectangular geometry consistent with the shape of our slit.

Integrating over the entire coma and our slit FOV allows us to calculate the aperture correction. We then calculate the production rate using:

$Q=\frac{4 \pi \Delta^{2} F A C}{g \tau}$

where $Q$ is the production rate, $\Delta$ is the geocentric distance, $F$ is the observed flux, $A C$ is the aperture correction, $g$ is the g-factor (fluorescence efficiency), and $\tau$ is the dissociative lifetime. The g-factors we employ are displayed in Table 2 . For $\mathrm{CN}$ and $\mathrm{C}_{2}$, we adopt g-factors from Schleicher (2010) and A'Hearn et al. (1995), respectively. For $\mathrm{NH}_{2}$, we derive our production rate using the $(0,5,0)$ band (bent notation) and employ g-factors from H. Kawakita (private communication), which were calculated using the same model as in Kawakita and Watanabe (2002). For $\mathrm{CH}$ we adopt the g-factor from Singh and Dalgarno (1987), although we include the Swings Effect, which we discuss in the next section.

One of the shortcomings of the Haser model is that it is time independent. This means that any short-term variability in production rates will cause deviations from the Haser profile. Therefore the column density along the line of sight will not necessarily be given by a Haser profile. This is potentially important for $103 \mathrm{P}$ because other investigations have observed short-term variability in production rates associated with the rotation of the nucleus (A'Hearn et al., 2011; Drahus et al., 2012). However, with our small 
Table 2

Parameter values used in the Haser models.

\begin{tabular}{|c|c|c|c|c|}
\hline Molecule & $\tau_{p}(\mathrm{~s})^{\mathrm{a}}$ & $\tau_{d}(\mathrm{~s})^{\mathrm{a}}$ & $V_{e j}\left(\mathrm{~km} \mathrm{~s}^{-1}\right)$ & g-Factor (ergs s ${ }^{-1}$ molecule $\left.^{-1}\right)^{a}$ \\
\hline $\mathrm{CN}$ & $1.3 \times 10^{4}$ & $2.1 \times 10^{5}$ & 1.02 & $2.6 \times 10^{-13}$ \\
\hline $\mathrm{CH}$ & $7.8 \times 10^{4}$ & $4.8 \times 10^{3}$ & 2.18 & $2.3 \times 10^{-14}$ \\
\hline $\mathrm{C} 2$ & $2.2 \times 10^{4}$ & $6.6 \times 10^{4}$ & 1.38 & $4.5 \times 10^{-13}$ \\
\hline $\mathrm{NH}_{2}$ & $4.1 \times 10^{3}$ & $6.2 \times 10^{4}$ & 1.13 & $6.40 \times 10^{-15}$ \\
\hline $\mathrm{OI}^{\mathrm{b}}$ & $8.3 \times 10^{4}$ & - & - & - \\
\hline $\mathrm{OI}^{\mathrm{c}}$ & $1.3 \times 10^{5}$ & - & - & - \\
\hline $\mathrm{OI}^{\mathrm{d}}$ & $5.0 \times 10^{5}$ & - & - & - \\
\hline
\end{tabular}

a Given for $r=1 \mathrm{AU}$. For CN and $\mathrm{CH}$, given for $\dot{r}=0 \mathrm{~km} \mathrm{~s}^{-1}$, but varies with $\dot{r}$ (see Schleicher (2010) for CN and Fig. 4 for $\mathrm{CH}$ ).

b For [OI] from dissociation of $\mathrm{H}_{2} \mathrm{O}$ into $\mathrm{H}_{2}$ and $\mathrm{O}$; branching ratio employed is 0.07 (Bhardwaj and Raghuram, 2012).

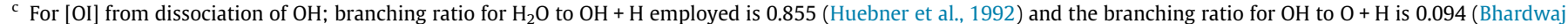
and Raghuram, 2012).

${ }^{d}$ For [OI] from dissociation of $\mathrm{CO}_{2}$ into $\mathrm{CO}+\mathrm{O}$; branching ratio employed is 0.72 (Bhardwaj and Raghuram, 2012).

projected slit size, most of the observed flux originates from the inscribed sphere, so any systematic error from the Haser model assumption introduced by the presence of short-term variability is small.

\subsection{Possible Swings Effect for $\mathrm{CH}$}

Due to the presence of $\mathrm{CH}$ absorption features in the solar spectrum, $\mathrm{CH}$ emission could be subject to a significant Swings Effect. The Swings Effect (Swings, 1941) is a variation in the g-factor due to the Doppler Shift induced by the heliocentric velocity of the comet. Depending on the heliocentric velocity, the exciting wavelength for the fluorescence transitions observed can be shifted into or out of absorption features in the solar spectrum. This can change the available flux to drive a given transition substantially. The Swings Effect is well documented for the CN $\Delta v=0$ transition (e.g. Schleicher, 2010).

We used the BASS2000 solar spectrum (http://bass2000.obspm.fr/solar_spect.php), which is highly variable as a function of wavelength near the $\mathrm{CH}$ A-X emission band, due to the presence of $\mathrm{CH}$ absorption in the solar spectrum (the so-called

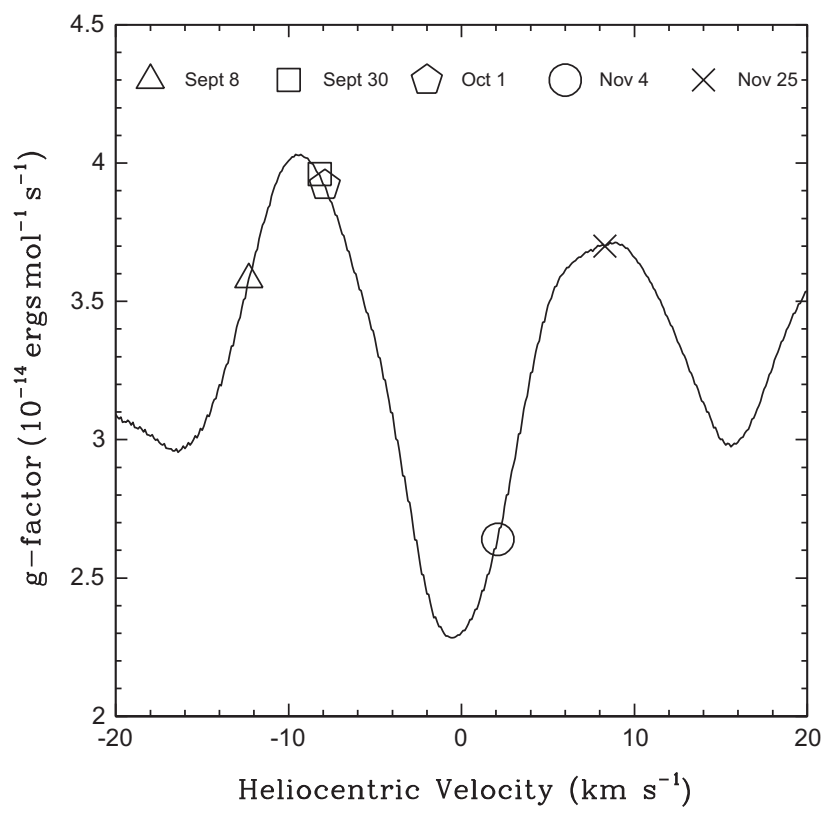

Fig. 4. Calculated g-factor for the $\mathrm{CH}$ A-X band as a function of heliocentric velocity. As expected, there is a strong minimum near zero heliocentric velocity. The g-factor is fairly symmetric with respect to perihelion (zero heliocentric velocity). Overplotted are symbols showing the heliocentric velocity and g-factor for 103P on each of our observing dates.
"G-band"). We plot the g-factor as a function of heliocentric velocity in Fig. 4. We calculated the g-factors by multiplying the g-factor we adopt from Singh and Dalgarno (1987) at a heliocentric velocity of zero $\mathrm{km} \mathrm{s}^{-1}\left(2.3 \times 10^{-14} \mathrm{ergs} \mathrm{s}^{-1} \mathrm{~mol}^{-1}\right)$ by the ratio of the solar flux available to pump the band at $\dot{r}=0 \mathrm{~km} \mathrm{~s}^{-1}$ with the solar flux at the heliocentric velocity of interest. There is a strong minimum near $\dot{r}=0 \mathrm{~km} \mathrm{~s}^{-1}$, as would be expected, but the g-factor is fairly symmetric between comparable positive and negative velocities. We adopt these updated g-factors when calculating the $\mathrm{CH}$ production rates. This is not a detailed fluorescence calculation, thus this is an approximation to the actual Swings effect. A detailed calculation is beyond the scope of this work, but will be the subject of future work.

\section{7. $\mathrm{H}_{2} \mathrm{O}$ production rate from [O I]6300 emission}

As molecular abundances in comets are typically calculated with respect to $\mathrm{H}_{2} \mathrm{O}$, it is desirable to have observations of $\mathrm{H}_{2} \mathrm{O}$ to compare to our derived molecular production rates. $\mathrm{H}_{2} \mathrm{O}$ does not have any transitions in the optical, but our bandpass includes the [O I] $6300 \AA$ line, which has been used as a reliable proxy for $\mathrm{H}_{2} \mathrm{O}$ production in the past (Spinrad, 1982; Magee-Sauer et al., 1990; Schultz et al., 1992; Morgenthaler et al., 2001; Morgenthaler et al., 2007; McKay et al., 2012). We employ the same Haser Model for the [OI] emission as used in McKay et al. (2012) to infer $\mathrm{H}_{2} \mathrm{O}$ production rates from our [OI] observations.

Since 103P has a large $\mathrm{CO}_{2}$ abundunce, we have included $\mathrm{CO}_{2}$ as a source of ${ }^{1} \mathrm{D}$ oxygen atoms. The $\mathrm{CO}_{2} / \mathrm{H}_{2} \mathrm{O}$ ratio varied from $10 \%$ to $20 \%$, depending on the rotational phase of the nucleus (A'Hearn et al., 2011). Therefore we have assumed a $\mathrm{CO}_{2} / \mathrm{H}_{2} \mathrm{O}$ ratio of 0.15 when estimating the contribution of $\mathrm{CO}_{2}$ to the ${ }^{1} \mathrm{D}$ oxygen atom population. For estimating the $\mathrm{CO}_{2}$ contribution, we use a very similar Haser Model to that used for $\mathrm{H}_{2} \mathrm{O}$. We find that for $\mathrm{CO}_{2} /$ $\mathrm{H}_{2} \mathrm{O}=0.15$, approximately $25 \%$ of the ${ }^{1} \mathrm{D}$ oxygen atoms are contributed by $\mathrm{CO}_{2}$ photodissociation. Since the $\mathrm{CO}_{2} / \mathrm{H}_{2} \mathrm{O}$ ratio for $103 \mathrm{P}$ was variable, adopting a constant value of 0.15 may introduce a systematic error in our $\mathrm{H}_{2} \mathrm{O}$ production rates. However, the contribution from $\mathrm{CO}_{2}$ ranges from $17 \%$ for $\mathrm{CO}_{2} / \mathrm{H}_{2} \mathrm{O}=0.1$ to $34 \%$ for $\mathrm{CO}_{2} /$ $\mathrm{H}_{2} \mathrm{O}=0.2$. Employing these $\mathrm{CO}_{2} / \mathrm{H}_{2} \mathrm{O}$ ratios rather than the average value of 0.15 changes the $\mathrm{H}_{2} \mathrm{O}$ production rates by less than $10 \%$, so adopting an average value of $\mathrm{CO}_{2} / \mathrm{H}_{2} \mathrm{O}$ does not affect our derived $\mathrm{H}_{2} \mathrm{O}$ production rates significantly.

\section{Results}

The resulting average production rates for each observation date are given in Table 3. The mixing ratios relative to $\mathrm{H}_{2} \mathrm{O}$ use the $\mathrm{H}_{2} \mathrm{O}$ production rate derived from the cometary [OI]6300 line (see Table 4). 
Table 3

Production rates.

\begin{tabular}{lcllll}
\hline UT Date & \multicolumn{2}{l}{$Q\left(10^{25} \mathrm{~mol} \mathrm{~s}^{-1}\right)$} & & \\
\cline { 2 - 6 } & $\mathrm{H}_{2} \mathrm{O}$ & $\mathrm{CN}$ & $\mathrm{CH}$ & $\mathrm{C}_{2}$ & $\mathrm{NH}_{2}$ \\
\hline $9 / 8 / 2010$ & $336 \pm 34$ & $0.41 \pm 0.04$ & $3.39 \pm 0.38$ & $0.54 \pm 0.05$ & $1.05 \pm 0.11$ \\
$9 / 30 / 2010$ & $556 \pm 56$ & $0.67 \pm 0.07$ & $3.98 \pm 0.40$ & $0.82 \pm 0.08$ & $1.62 \pm 0.16$ \\
$10 / 1 / 2010$ & $804 \pm 80$ & $1.27 \pm 0.13$ & $7.42 \pm 0.74$ & $1.24 \pm 0.12$ & $2.79 \pm 0.28$ \\
$11 / 04 / 2010$ & $1030 \pm 110$ & $1.46 \pm 0.15$ & $5.18 \pm 0.52$ & $1.47 \pm 0.15$ & $2.76 \pm 0.28$ \\
$11 / 26 / 2010$ & $896 \pm 90$ & $2.78 \pm 0.28$ & $8.45 \pm 1.37$ & $2.02 \pm 0.20$ & $4.03 \pm 0.40$ \\
\hline
\end{tabular}

\subsection{Rotational variation}

We observe variability in gas production on hour timescales, which is presumably associated with the rotation of the nucleus. This variability also has been inferred by other studies for parent molecules at IR (Dello Russo et al., 2011; Mumma et al., 2011) and sub-mm (Drahus et al., 2012) wavelengths. We show a plot of production rate vs. time for CN on UT September 30-October 1 in Fig. 5, and the same for $\mathrm{C}_{2}, \mathrm{CH}, \mathrm{NH}_{2}$, and $\mathrm{H}_{2} \mathrm{O}$ in Fig. 6. This variability is not consistent with a simple sinusoid function, but rather a juxtaposition of two rotational phases, namely the $\sim 18 \mathrm{~h}$ precession period and the $\sim 27 \mathrm{~h}$ "roll". Our production rate measurements on UT September 30 and UT October 1 are fit fairly well by a sum of two sinusoids with the precession and "roll" periods found by other studies (A'Hearn et al., 2011; Samarasinha et al., 2011; Knight and Schleicher, 2011; Belton et al., 2013; Knight and Schleicher, 2013). The functional fits are overplotted on the data shown in Figs. 5 and 6.

We derived the fits using the IDL routine MPFIT (Markwardt, 2009), which employs a $\chi$-squared minimization technique. The fits for the periodicity are a sum of two sine waves with periods of 17.6 and $29.0 \mathrm{~h}$ (the periods for the precession and roll found by Belton et al. (2013) at the time of these observations). We kept these periods as fixed parameters during the fitting process to limit the number of free parameters. The phase was kept a free parameter for the fit to the absolute production rate of $\mathrm{CN}$. The retrieved phase is very sensitive to the initial value, so we had to try several iterations with different phases in order to arrive at a satisfactory fit. Once we found the phase of the variation in the $\mathrm{CN}$ production rate, we fixed the phase for subsequent fits to the absolute production rates and mixing ratios. There is no evidence for a significant phase difference among species, consistent with other studies (A'Hearn et al., 2011; Drahus et al., 2012). The sum of the two rotational modes results in a quasi-periodic function with a period of $\sim 55 \mathrm{~h}$, similar to the periodicity found from studies of the CN morphology (Samarasinha et al., 2011; Knight and Schleicher, 2011). However, our data set does not consist of a continuous temporal baseline long enough to show this conclusively, and only shows that our measurements are consistent with this interpretation.

An alternative way to determine whether we are observing actual variation in gas production is to calculate the standard deviation of our production rates and compare that to the average uncertainty associated with the measured production rates. If the

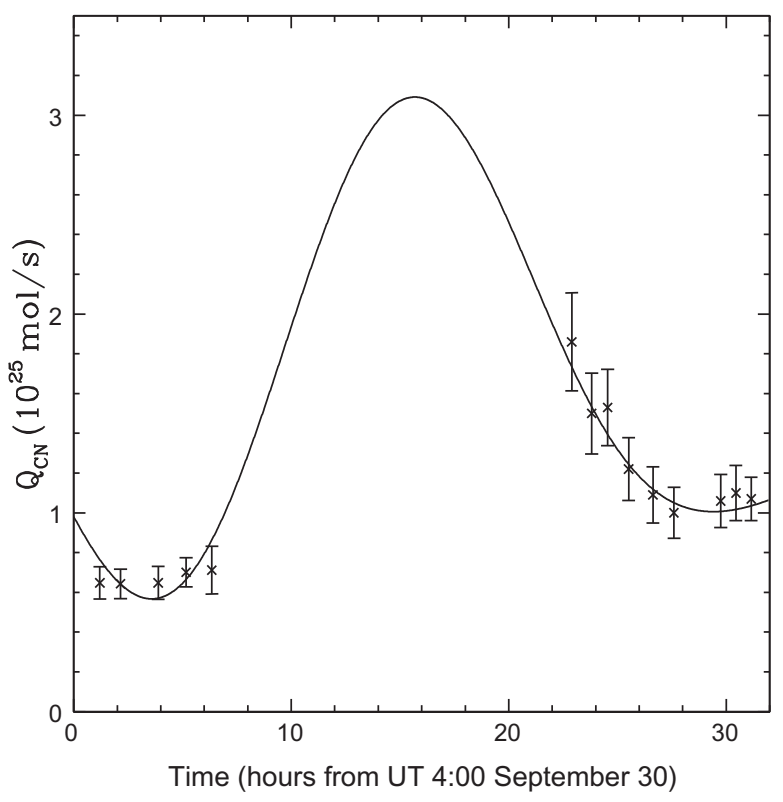

Fig. 5. Production rate vs. time for $\mathrm{CN}$ on UT September 30-October 1 . The gap in the data is due to daylight when we were unable to observe the comet. The functional fit to the data is overplotted as a solid line.

standard deviation is much higher than the average uncertainty, then there is evidence for variability in the gas production rates, which in the case of 103P is likely due to rotational modulation. We show the average gas production rates over the apparition, the average uncertainty in the production rates, and the standard deviation of the production rates for each species in Table 5 (we have excluded the September 8 observations to avoid introducing possible variation due to changing heliocentric distance). All species have a standard deviation in their production rates that is at least three times greater than the average uncertainty, as shown in the last column of Table 5. Therefore it is likely that we have detected rotational modulation of the gas production rates.

Fig. 7 shows the mixing ratios $\mathrm{CN} / \mathrm{H}_{2} \mathrm{O}, \mathrm{C}_{2} / \mathrm{H}_{2} \mathrm{O}, \mathrm{CH} / \mathrm{H}_{2} \mathrm{O}$, and $\mathrm{NH}_{2} / \mathrm{H}_{2} \mathrm{O}$ for our UT September 30 and UT October 1 observations, while Fig. 8 shows $\mathrm{C}_{2} / \mathrm{CN}, \mathrm{CH} / \mathrm{CN}$, and $\mathrm{NH}_{2} / \mathrm{CN}$ on those same dates. Functional fits similar to those plotted in Figs. 5 and 6 are overplotted. In all cases, a periodic function can be fit to the data. However, we require that the inferred amplitude of the variation be at least twice the error bars to be considered a real variation. When this criterion is enforced, only the $\mathrm{CN} / \mathrm{H}_{2} \mathrm{O}$ (Fig. 7) and $\mathrm{C}_{2} / \mathrm{CN}$ (Fig. 8) ratios vary significantly. For all other mixing ratios, we plot a fit with constant mixing ratio, which in effect is the weighted mean. There may be variations in these mixing ratios, but our data do not have the signal to noise to show them definitively. Like the absolute production rates on September 30 and October 1, the $\mathrm{C}_{2} / \mathrm{CN}$ and the $\mathrm{CN} / \mathrm{H}_{2} \mathrm{O}$ ratio are also fit well by a sum of two sinusoids with the precession and "roll" periods found for 103P. The functional fit suggests that the $C_{2} / C N$ ratio varies from 0.7 to 1.3 and the $C N /$

Table 4

Mixing ratios.

\begin{tabular}{|c|c|c|c|c|c|c|c|}
\hline \multirow[t]{2}{*}{ UT Date } & \multicolumn{4}{|c|}{$\mathrm{X} / \mathrm{H}_{2} \mathrm{O} * 100.0$} & \multicolumn{3}{|l|}{$\mathrm{X} / \mathrm{CN}$} \\
\hline & $\mathrm{CN}$ & $\mathrm{C}_{2}$ & $\mathrm{CH} \mathrm{NH} 2$ & & $\mathrm{C}_{2}$ & $\mathrm{CH}$ & $\mathrm{NH}_{2}$ \\
\hline $9 / 8 / 2010$ & $0.12 \pm 0.01$ & $0.16 \pm 0.01$ & $1.01 \pm 0.11$ & $0.31 \pm 0.03$ & $1.32 \pm 0.12$ & $8.32 \pm 1.14$ & $2.58 \pm 0.31$ \\
\hline $9 / 30 / 2010$ & $0.12 \pm 0.01$ & $0.15 \pm 0.01$ & $0.72 \pm 0.06$ & $0.29 \pm 0.02$ & $1.22 \pm 0.05$ & $5.94 \pm 0.51$ & $2.42 \pm 0.15$ \\
\hline $10 / 1 / 2010$ & $0.16 \pm 0.01$ & $0.15 \pm 0.01$ & $0.93 \pm 0.04$ & $0.35 \pm 0.01$ & $0.97 \pm 0.03$ & $5.83 \pm 0.31$ & $2.19 \pm 0.10$ \\
\hline $11 / 4 / 2010$ & $0.14 \pm 0.01$ & $0.14 \pm 0.01$ & $0.50 \pm 0.05$ & $0.27 \pm 0.03$ & $1.01 \pm 0.05$ & $3.55 \pm 0.32$ & $1.89 \pm 0.18$ \\
\hline $11 / 26 / 2010$ & $0.31 \pm 0.04$ & $0.22 \pm 0.02$ & $0.94 \pm 0.18$ & $0.45 \pm 0.03$ & $0.72 \pm 0.05$ & $3.04 \pm 0.52$ & $1.45 \pm 0.15$ \\
\hline
\end{tabular}




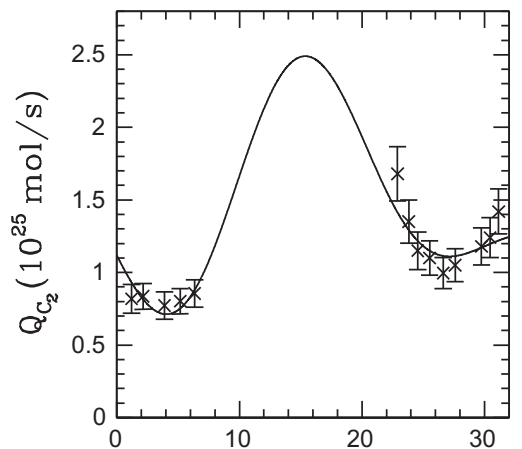

Time (hours from UT 4:00 September 30)

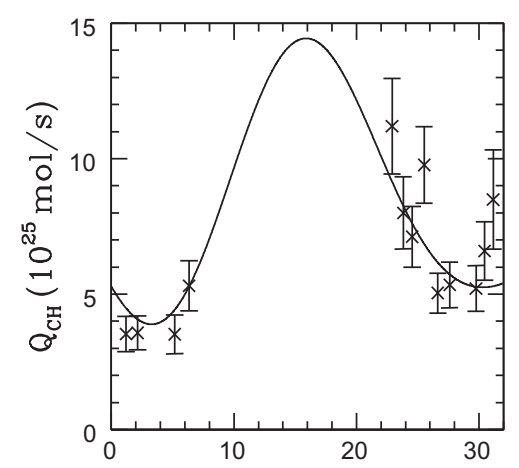

Time (hours from UT 4:00 September 30)

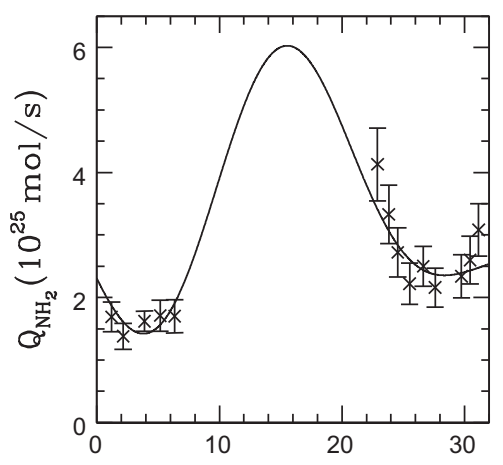

Time (hours from UT 4:00 September 30)

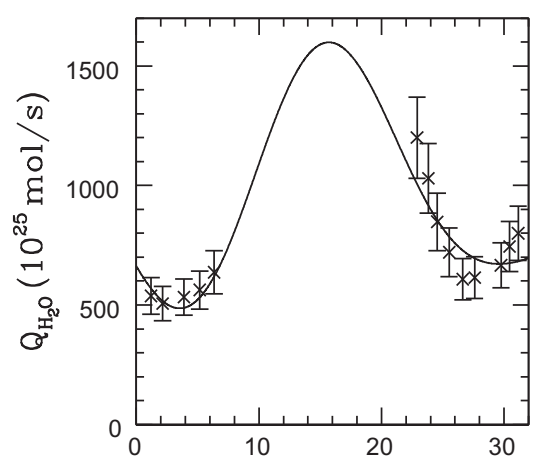

Time (hours from UT 4:00 September 30)

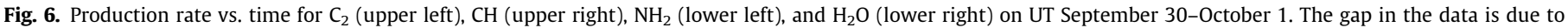
daylight when we were unable to observe the comet. Functional fits to the data are overplotted as solid lines.

Table 5

Average production rates and standard deviation. ${ }^{a}$

\begin{tabular}{|c|c|c|c|c|c|c|}
\hline Species & Ave. $Q^{\mathrm{b}}$ & Ave. $\sigma^{\mathrm{b}}$ & Ave. $\sigma$ (\% of ave.) & Standard dev. ${ }^{\text {b }}$ & Standard dev. (\% of ave.) & Standard dev./ave. $\sigma$ \\
\hline $\mathrm{CN}$ & 1.22 & 0.12 & 10 & 0.55 & 45 & 4.5 \\
\hline $\mathrm{CH}$ & 6.27 & 0.85 & 14 & 2.3 & 37 & 2.6 \\
\hline $\mathrm{NH}_{2}$ & 2.53 & 0.26 & 10 & 0.84 & 33 & 3.3 \\
\hline $\mathrm{C}_{2}$ & 1.20 & 0.12 & 10 & 0.39 & 33 & 3.3 \\
\hline $\mathrm{H}_{2} \mathrm{O}$ & 777 & 80 & 10 & 260 & 33 & 3.3 \\
\hline
\end{tabular}

a Excludes values from UT September 8 to avoid possible heliocentric distance variations.

b In units of $10^{25} \mathrm{~mol} \mathrm{~s}^{-1}$.

$\mathrm{H}_{2} \mathrm{O}$ ratio varies from 0.12 to 0.24 , but as is the case with the inferred functional form of the variation of the absolute gas production rates, analysis of our data set does not provide a conclusive finding and is only consistent with the proposed scenario.

We present the average mixing ratios, average uncertainties, and standard deviations in Table 6. Applying the criterion that the ratio of the standard deviation to the average uncertainty be greater than two (see last column of Table 6) in order for a mixing ratio to be considered variable, we determine that most mixing ratios are fairly constant. The only ones that vary significantly are $\mathrm{CN} / \mathrm{H}_{2} \mathrm{O}$ and $\mathrm{C}_{2} / \mathrm{CN}$, which are the same ratios inferred to be variable from the period fitting approach. Therefore we have evidence from two independent approaches that the $\mathrm{CN} / \mathrm{H}_{2} \mathrm{O}$ and $\mathrm{C}_{2} / \mathrm{CN}$ abundance ratios for our observations of 103P exhibit significant variability. A similarly variable $\mathrm{CN} / \mathrm{H}_{2} \mathrm{O}$ ratio with rotational phase is also observed in the DIXI flyby data (D. Bodewits, private communication and in prep.). It is possible other mixing ratios also exhibit variability, but analysis of our data does not definitively demonstrate this. Comparison with other data sets provides possible evidence that $\mathrm{NH}_{2} / \mathrm{H}_{2} \mathrm{O}$ is variable (see Section 4.1).

Fig. 6 suggests that $\mathrm{CH}$ (top right) and $\mathrm{NH}_{2}$ (bottom left) experience strong rotational modulation in their gas production rates, but not quite as strong as $\mathrm{CN}$ (Fig. 5). $\mathrm{C}_{2}$ shows a much weaker dependence on rotational phase of the nucleus (top left of Fig. 6), and $\mathrm{H}_{2} \mathrm{O}$ shows the weakest rotational modulation (bottom right of Fig. 6), though the variation is still a factor of 3 (this relative hierarchy, with $\mathrm{CN}$ exhibiting the most variation and $\mathrm{C}_{2}$ and $\mathrm{H}_{2} \mathrm{O}$ the least, is also supported by examination of the standard deviations, see Table 5). The stark difference in the amount of rotational modulation for $\mathrm{C}_{2}$ and $\mathrm{H}_{2} \mathrm{O}$ as compared to $\mathrm{CN}$ is manifested in the changing mixing ratios of these species displayed in Figs. 7 and 8 and Table 6 , while for $\mathrm{NH}_{2}$ and $\mathrm{CH}$ the data are too noisy to see a definitive change in mixing ratios compared to either $\mathrm{CN}$ or $\mathrm{H}_{2} \mathrm{O}$ with rotational phase.

\subsection{Changes in mixing ratios pre- vs. post-perihelion}

We plot the mixing ratios relative to $\mathrm{H}_{2} \mathrm{O}$ and $\mathrm{CN}$ over the course of the apparition in Figs. 11 and 12, respectively. Most of the variation in mixing ratios can be attributed to rotational modulation. However, the $\mathrm{CH} / \mathrm{CN}$ ratio is much higher pre-perihelion than post-perihelion, and it seems it cannot be attibuted to rotational variation (compare with the range of observed $\mathrm{CH} / \mathrm{CN}$ values in Fig. 8). Examination of the production rates shows that the 


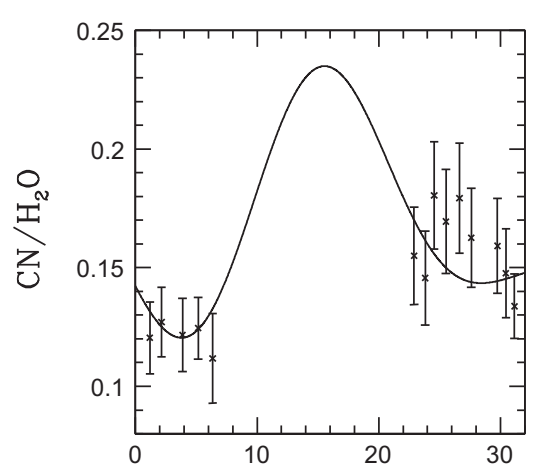

Time (hours from UT 4:00 September 30)

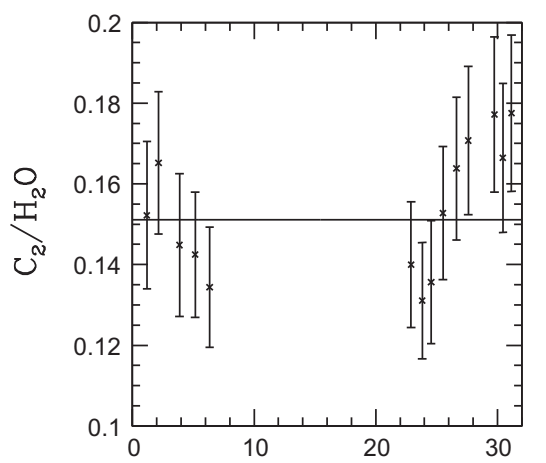

Time (hours from UT 4:00 September 30)

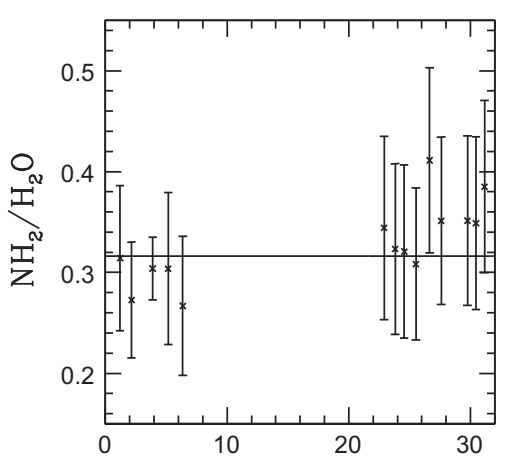

Time (hours from UT 4:00 September 30)

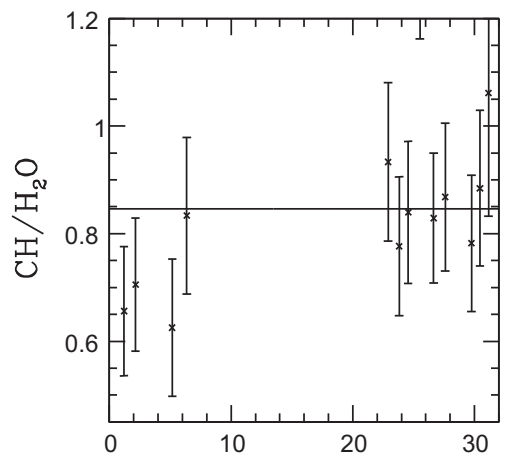

Time (hours from UT 4:00 September 30)

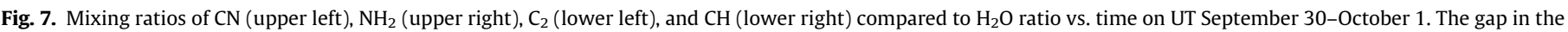

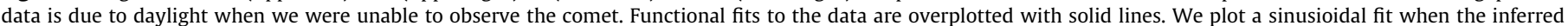

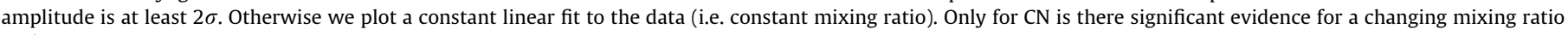
with respect to $\mathrm{H}_{2} \mathrm{O}$.
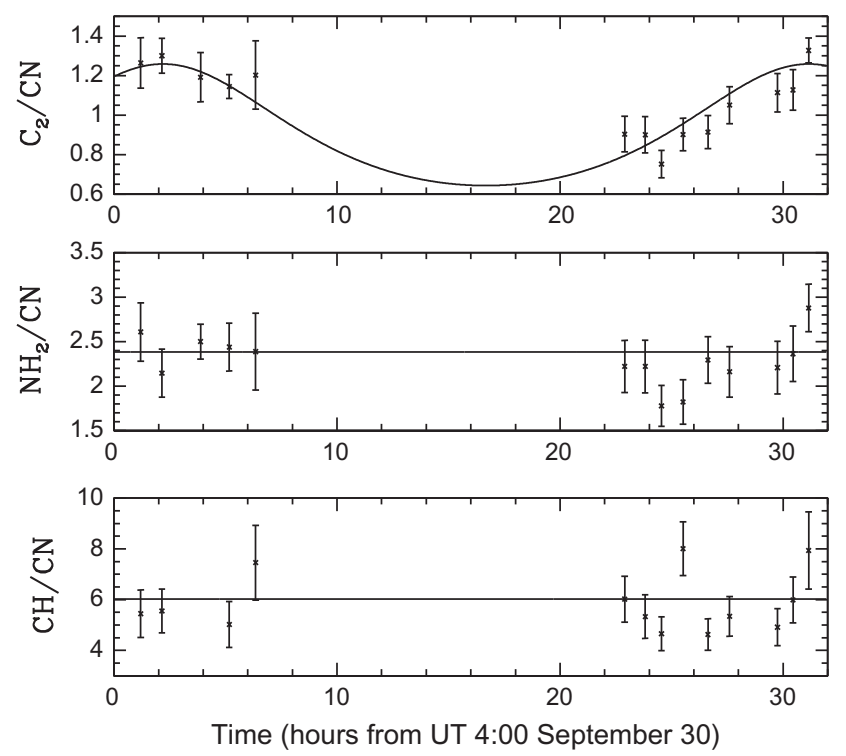

Fig. 8. Mixing ratios of $\mathrm{C}_{2}$ (top), $\mathrm{NH}_{2}$ (middle), and $\mathrm{CH}$ (bottom) relative to $\mathrm{CN}$ vs. time on UT September 30-October 1 . The gap in the data is due to daylight when we were unable to observe the comet. Functional fits to the data are overplotted with solid lines. The same criterion of plotting a sinusoid or constant linear fit in Fig. 7 applies here. Only $\mathrm{C}_{2} / \mathrm{CN}$ shows significant evidence for variability over a rotation.

production rate of $\mathrm{CH}$ remains fairly constant, while that of $\mathrm{CN}$ and other molecules is higher post-perihelion. This suggests that $\mathrm{CN}$ and other species may have experienced an increase in production rates post-perihelion (this has also been observed by Knight and Schleicher (2013)), while CH did not.

\section{Discussion}

\subsection{Comparison to other studies}

Our production rates for $\mathrm{CN}$ and $\mathrm{C}_{2}$ are within $20 \%$ of those found by Knight and Schleicher (2013) for dates where our observations are separated by less than a day from theirs (i.e. September 8 , September 30, and October 1 ). Our inferred $\mathrm{H}_{2} \mathrm{O}$ production rates from [OI]6300 observations on these dates are in agreement with the $\mathrm{H}_{2} \mathrm{O}$ production rates inferred from $\mathrm{OH}$ observations by Knight and Schleicher (2013) and Lyman- $\alpha$ observations by Combi et al. (2011). Though they did not observe $\mathrm{NH}_{2}$, Knight and Schleicher (2013) report $\mathrm{NH}$ production rates. Since $\mathrm{NH}_{2}$ and $\mathrm{NH}$ are both thought to be released into the coma via photodissociation of $\mathrm{NH}_{3}\left(\mathrm{NH}_{2}\right.$ directly, $\mathrm{NH}$ through a two step process involving $\mathrm{NH}_{2}$ ), the production rates for $\mathrm{NH}_{2}$ and $\mathrm{NH}$ should be similar. Our $\mathrm{NH}_{2}$ production rates are significantly lower (50\%) than the $\mathrm{NH}$ production rates determined by Knight and Schleicher (2013). This may mean there is an additional source of $\mathrm{NH}$ in 103P's coma (see Section 4.3 for more discussion on this possibility).

Our production rates on November 4 are all much lower than those found by Shinnaka et al. (2013). However, we infer that our observations occurred just before a minimum in the gas production. The observations by Shinnaka et al. (2013), which occurred about $9 \mathrm{~h}$ (half a rotation) after ours, would have sampled the comet near a maximum in gas activity. If this is correct, then the disrepency can be explained by the observations being at 
Table 6

Average mixing ratios and standard deviation.

\begin{tabular}{|c|c|c|c|c|c|c|}
\hline Mixing ratio & Ave. & Ave. $\sigma$ & Ave. $\sigma$ (\% of ave.) & Standard dev. & Standard dev. (\% of ave.) & Standard dev./ave. $\sigma$ \\
\hline $\mathrm{CN} / \mathrm{H}_{2} \mathrm{O}^{\mathrm{a}}$ & 0.16 & 0.019 & 12 & 0.046 & 29 & 2.4 \\
\hline $\mathrm{CH} / \mathrm{H}_{2} \mathrm{O}^{\mathrm{a}}$ & 0.84 & 0.14 & 17 & 0.19 & 23 & 1.4 \\
\hline $\mathrm{NH}_{2} / \mathrm{H}_{2} \mathrm{O}^{\mathrm{a}}$ & 0.32 & 0.033 & 10 & 0.051 & 16 & 1.6 \\
\hline $\mathrm{C}_{2} / \mathrm{H}_{2} \mathrm{O}^{\mathrm{a}}$ & 0.16 & 0.017 & 11 & 0.023 & 14 & 1.4 \\
\hline $\mathrm{C}_{2} / \mathrm{CN}$ & 1.03 & 0.08 & 8 & 0.17 & 17 & 2.1 \\
\hline $\mathrm{CH} / \mathrm{CN}$ & 5.69 & 0.85 & 15 & 1.05 & 18 & 1.2 \\
\hline $\mathrm{NH}_{2} / \mathrm{CN}$ & 2.13 & 0.27 & 13 & 0.31 & 15 & 1.2 \\
\hline
\end{tabular}

a Values given in $\%$.

different rotational phases. Observations by Dello Russo et al. (2011) nearly bridge the gap between our observations and those of Shinnaka et al. (2013). We present a plot of gas production for $\mathrm{H}_{2} \mathrm{O}, \mathrm{CN} / \mathrm{HCN}$, and $\mathrm{NH}_{2} / \mathrm{NH}_{3}$ on November 4 from this work, Dello Russo et al. (2011), and Shinnaka et al. (2013) in Fig. 9. Gas production increased throughout the course of the Dello Russo et al. (2011) observations, supporting the conclusion that differences in rotational phase for the observations is responsible for the discrepency in gas production rates. Also, the peak in $\mathrm{HCN}$ and $\mathrm{CH}_{3} \mathrm{OH}$ production determined by Drahus et al. (2012) occurred just after the Shinnaka et al. (2013) observations (we employ the same rotational phase convention as Drahus et al. (2012) and Shinnaka et al. (2013)), again suggesting that their observations occurred during maximum gas production. Similarly, the minimum of $\mathrm{HCN}$ and $\mathrm{CH}_{3} \mathrm{OH}$ production observed by Drahus et al. (2012) occurs at a rotational phase of 0.3 , the same as our minimum in gas production.

We plot the mixing ratios of $\mathrm{NH}_{2} / \mathrm{NH}_{3}$ and $\mathrm{CN} / \mathrm{HCN}$ compared to $\mathrm{H}_{2} \mathrm{O}$ on November 4 from Dello Russo et al. (2011), Shinnaka et al. (2013), and this work in Fig. 10. There is evidence that $\mathrm{CN} / \mathrm{H}_{2} \mathrm{O}$ changed with rotational phase. The highest mixing ratios happen to occur at peaks in the gas production rate (compare Figs. 9 and

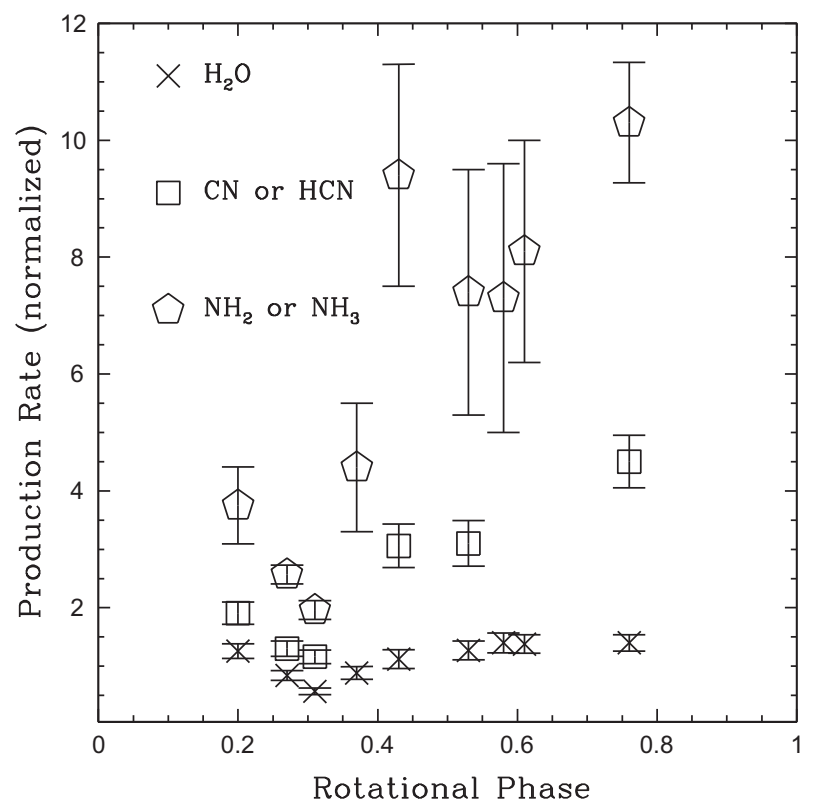

Fig. 9. $\mathrm{H}_{2} \mathrm{O}, \mathrm{CN}$ or $\mathrm{HCN}$, and $\mathrm{NH}_{2}$ or $\mathrm{NH}_{3}$ production rate on November 4 from Shinnaka et al. (2013) (last point, rotational phase 0.75), this work (first three points, rotational phase 0.2-0.35), and Dello Russo et al. (2011)(all remaining data points between rotational phase $0.35-0.65)$. Production rates from Dello Russo et al. (2011) are for $\mathrm{H}_{2} \mathrm{O}, \mathrm{NH}_{3}$, and $\mathrm{HCN}$, while production rates from Shinnaka et al. (2013) and this work are for $\mathrm{H}_{2} \mathrm{O}, \mathrm{NH}_{2}$, and $\mathrm{CN}$. Note the increase in gas production over the course of the observations, likely due to rotational modulation. We normalize the production rates so that they all display clearly on the same plot.
10). For $\mathrm{CN}$ on October 1 , the peak $\mathrm{CN} / \mathrm{H}_{2} \mathrm{O}$ ratio corresponds to higher production rates (compare Fig. 5 and top left panel of Fig. 7). This comparison also provides evidence that the $\mathrm{NH}_{2} / \mathrm{H}_{2} \mathrm{O}$ ratio changes over the course of the observations, but does have the caveat that we are comparing different observations with different observing geometries and observing different transitions of $\mathrm{NH}_{2}$ and $\mathrm{NH}_{3}$.

Our measurements of the $\mathrm{C}_{2} / \mathrm{CN}$ ratio are lower than that measured by other studies for 103P. However, all previous measurements were done using either low resolution spectra or narrowband photometry. Although extensive methods have been developed to minimize contamination of these types of observations by the continuum or other gas species (e.g. Farnham et al., 2000), there is some level of contamination present. This has the greatest effect for $C_{2}$, for which the wavelength region traditionally employed as the $\mathrm{C}_{2} \Delta v=0$ band also includes unidentified emission and $\mathrm{NH}_{2}$ lines. Our high spectral resolution observations isolate these lines and our fitting routines more accurately establish the flux from each molecular band.

For our 103P observations, the contaminating flux from unidentified emission and $\mathrm{NH}_{2}$ is about $30-40 \%$ that of the $\mathrm{C}_{2}$ flux. When this contaminating flux is included as $\mathrm{C}_{2}$ flux, our $\mathrm{C}_{2} / \mathrm{CN}$ ratios are

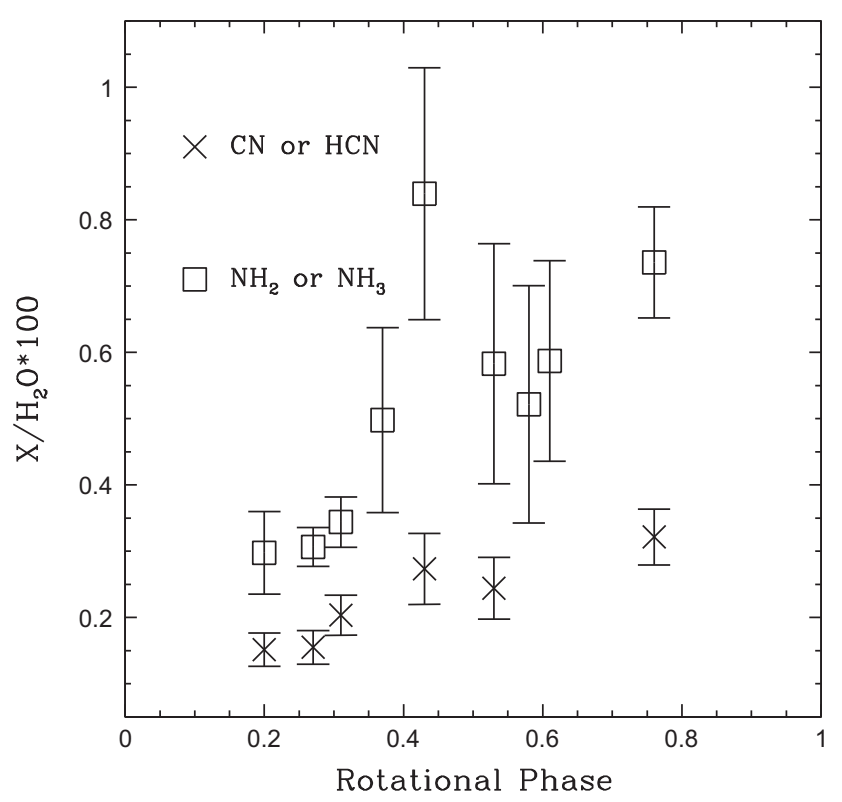

Fig. 10. Mixing ratios of $\mathrm{CN}$ or $\mathrm{HCN}$ and $\mathrm{NH}_{2}$ or $\mathrm{NH}_{3}$ compared to $\mathrm{H}_{2} \mathrm{O}$ vs. time on UT November 4 . The first three data points (between rotational phase 0.2 and 0.35 ) are from this work and are measurements of $\mathrm{CN} / \mathrm{H}_{2} \mathrm{O}$ and $\mathrm{NH}_{2} / \mathrm{H}_{2} \mathrm{O}$. The last data point at rotational phase 0.75 is from Shinnaka et al. (2013) and is also a measurement of $\mathrm{CN} / \mathrm{H}_{2} \mathrm{O}$ and $\mathrm{NH}_{2} / \mathrm{H}_{2} \mathrm{O}$. The rest of the data points in between rotational phase 0.35 and 0.65 are from Dello Russo et al. (2011) and are measurements of $\mathrm{HCN} / \mathrm{H}_{2} \mathrm{O}$ and $\mathrm{NH}_{3} / \mathrm{H}_{2} \mathrm{O}$. Here we observe evidence that both $\mathrm{CN} / \mathrm{H}_{2} \mathrm{O}$ and $\mathrm{NH}_{2} / \mathrm{H}_{2} \mathrm{O}$ change with rotational phase of the nucleus. 


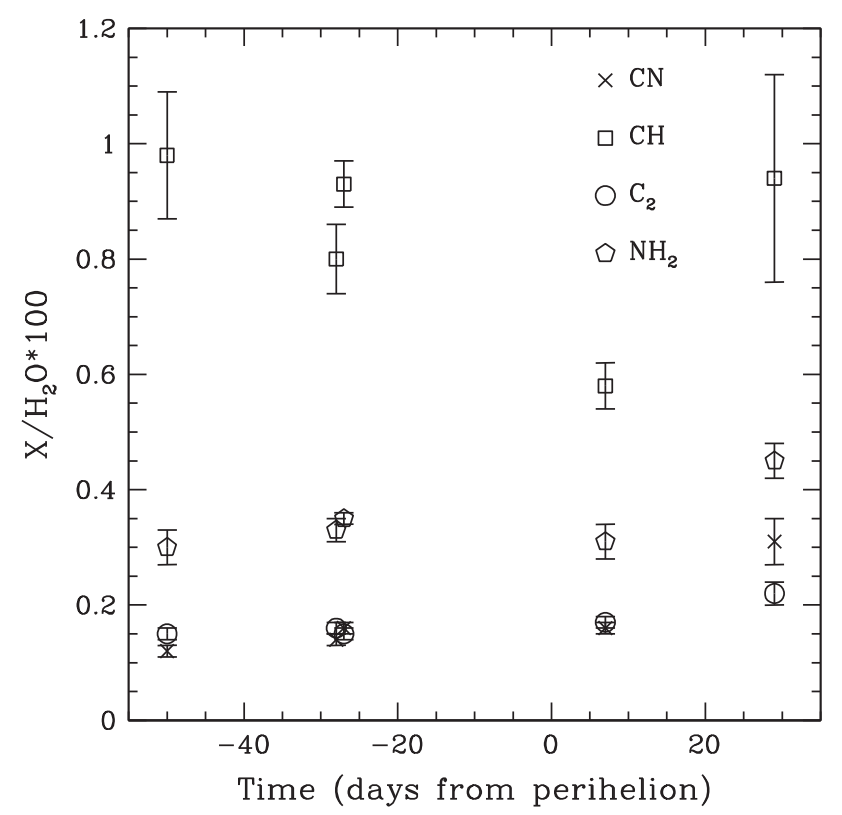

Fig. 11. Mixing ratios relative to $\mathrm{H}_{2} \mathrm{O}$ throughout the apparition. All the observed variation can be explained as rotational modulation. Therefore there is no evidence for a significant change in mixing ratios compared to $\mathrm{H}_{2} \mathrm{O}$ over the apparition.

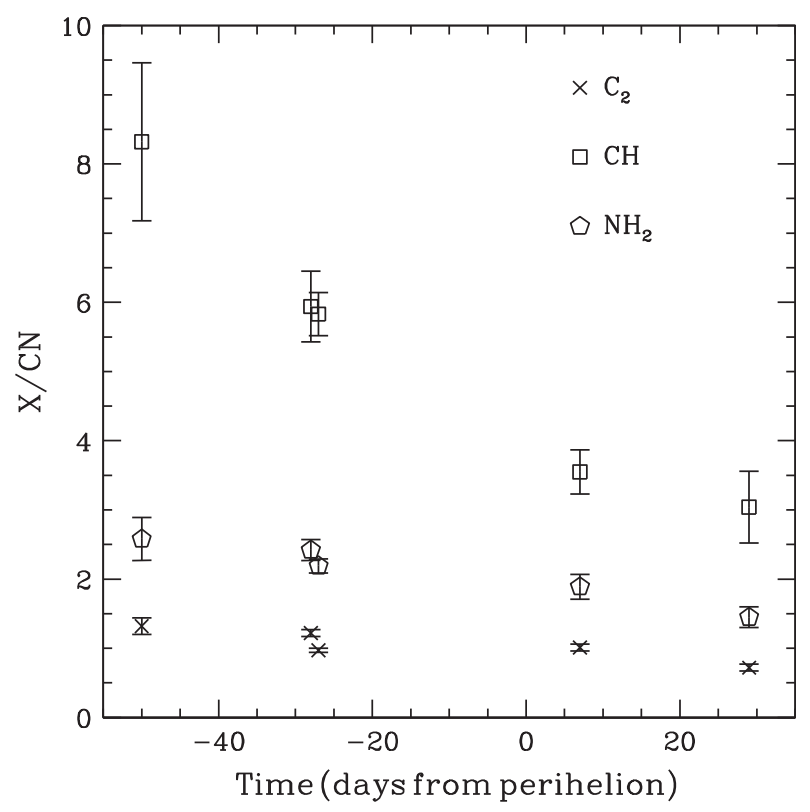

Fig. 12. Mixing ratios relative to $\mathrm{CN}$ throughout the apparition. The observed variation can be explained as rotational modulation, but the change in $\mathrm{CH} / \mathrm{CN}$ is too large to be explained by rotational modulation alone. Therefore we conclude that the $\mathrm{CH} / \mathrm{CN}$ ratio is higher pre-perihelion than post-perihelion.

consistent with those previously measured for 103P. This implies that $\mathrm{C}_{2} / \mathrm{CN}$ ratios measured from low resolution spectroscopy or narrowband photometry may not reflect the true abundunce ratio in cometary comae, which has implications when comparing the abundance of $\mathrm{C}_{2}$ to that of candidate parent molecules such as $\mathrm{C}_{2} \mathrm{H}_{2}$. It is also possible that $103 \mathrm{P}$ is overabundant in whatever species are responsible for the unidentified emission lines present in optical spectra of comets $\left(\mathrm{NH}_{2}\right.$ is not particularly overabundant in 103P compared to other comets, and is responsible for only a small fraction of the contaminating flux), and that for the majority of comets this is less of an issue. Moreover, due to the shorter scale length of $\mathrm{NH}_{2}$ compared to $\mathrm{C}_{2}, \mathrm{NH}_{2}$ contamination is much smaller for long slit spectroscopy, meaning that any contamination is likely from unidentified emission. More high spectral resolution optical observations of comets are needed to quantify the amount of unidentified line and $\mathrm{NH}_{2}$ emission present. A more detailed fluorescence model for these species that includes line-by-line g-factors would better predict how much contamination should be present. Detailed study of the unidentified emission features that leads to their identification would also help quantify the amount of contamination present and how this contamination may affect low spectral resolution observations.

\subsection{Comparison to candidate parent molecules}

We compare our measured abundunces of $\mathrm{CN}, \mathrm{C}_{2}, \mathrm{CH}$, and $\mathrm{NH}_{2}$ (averaged over the apparition) to candidate parent molecules observed at NIR wavelengths (Dello Russo et al., 2010, 2011; Mumma et al., 2011; Kawakita et al., 2013) (average of all measurements) in Table 7. Our $\mathrm{CN} / \mathrm{H}_{2} \mathrm{O}$ ratios are consistent with the $\mathrm{HCN} / \mathrm{H}_{2} \mathrm{O}$ ratios measured by NIR studies, suggesting that $\mathrm{HCN}$ is the primary parent of $\mathrm{CN}$ in the case of 103P. We measure a variability in the $\mathrm{CN} /$ $\mathrm{H}_{2} \mathrm{O}$ abundunce that does not seem to be present in the NIR observations of HCN. However, none of the IR observations have a long temporal baseline such as our UT September 30-October 1 observations, which sample the rotational modulation with baselines of hours.

The measured $\mathrm{C}_{2} / \mathrm{H}_{2} \mathrm{O}$ ratios are higher than the $\mathrm{C}_{2} \mathrm{H}_{2} / \mathrm{H}_{2} \mathrm{O}$ ratios measured for $103 \mathrm{P}$, which suggests the need for an additional source of $\mathrm{C}_{2}$ besides $\mathrm{C}_{2} \mathrm{H}_{2}$. $\mathrm{C}_{2} \mathrm{H}_{6}$ could account for the rest of the $\mathrm{C}_{2}$, but $\mathrm{C}_{2} \mathrm{H}_{6}$ is thought to have a low branching ratio for releasing $\mathrm{C}_{2}$, making it a poor candidate for $\mathrm{C}_{2}$ parentage (Weiler, 2012). In addition, the $\mathrm{C}_{2} \mathrm{H}_{6}$ spatial distribution is similar to those of HCN and $\mathrm{C}_{2} \mathrm{H}_{2}$. Since $\mathrm{HCN}$ and $\mathrm{C}_{2} \mathrm{H}_{6}$ have similar spatial distributions, it would be expected that $\mathrm{C}_{2}$ originating from $\mathrm{C}_{2} \mathrm{H}_{6}$ photodissociation would still have a rotational modulation similar in amplitude to $\mathrm{CN}$ (which likely is released via HCN photodissociation), which is not observed. Therefore, an as-of-yet unobserved hydrocarbon or CHON (carbonaceous dust) particles likely contribute significantly to the $\mathrm{C}_{2}$ population.

The $\mathrm{NH}_{2} / \mathrm{H}_{2} \mathrm{O}$ ratios we measure are less than the $\mathrm{NH}_{3} / \mathrm{H}_{2} \mathrm{O}$ ratios measured in the NIR, implying that no source other than $\mathrm{NH}_{3}$ is required. The lower $\mathrm{NH}_{2} / \mathrm{H}_{2} \mathrm{O}$ ratios compared to $\mathrm{NH}_{3} / \mathrm{H}_{2} \mathrm{O}$ may be due to the possible rotational modulation discussed in the previous section. The $\mathrm{CH} / \mathrm{H}_{2} \mathrm{O}$ ratio is higher than the upper limit for $\mathrm{CH}_{4} /$ $\mathrm{H}_{2} \mathrm{O}$ found by Dello Russo et al. (2010), suggesting that $\mathrm{CH}_{4}$ is not the only source of $\mathrm{CH}$. As is the case for $\mathrm{C}_{2}$, this may suggest an as-of-yet undetected hydrocarbon or CHON grains are contributing to the $\mathrm{CH}$ population in the coma of 103P. If the production of CHON particles did not increase post-perihelion like the gaseous species (and $\mathrm{CHON}$ particles are a significant source of $\mathrm{CH}$ ), this could explain the asymmetry in the $\mathrm{CH} / \mathrm{CN}$ ratio with respect to perihelion.

Table 7

Abundances of daughter species and candidate parent species.

\begin{tabular}{llll}
\hline Daughter & $\begin{array}{l}\text { Daughter abundance } \\
\left(\mathrm{X} / \mathrm{H}_{2} \mathrm{O} * 100\right)\end{array}$ & Parent & $\begin{array}{l}\text { Parent abundance } \\
\left(\mathrm{X} / \mathrm{H}_{2} \mathrm{O} * 100\right)\end{array}$ \\
\hline $\mathrm{CN}$ & $0.16 \pm 0.005$ & $\mathrm{HCN}$ & $0.24 \pm 0.01$ \\
$\mathrm{C}_{2}$ & $0.16 \pm 0.004$ & $\mathrm{C}_{2} \mathrm{H}_{2}$ & $0.11 \pm 0.01$ \\
$\mathrm{C}_{2}$ & $0.16 \pm 0.004$ & $\mathrm{C}_{2} \mathrm{H}_{6}$ & $0.75 \pm 0.02$ \\
$\mathrm{CH}$ & $0.84 \pm 0.03$ & $\mathrm{CH}_{4}$ & $<0.5$ \\
$\mathrm{NH}_{2}$ & $0.32 \pm 0.01$ & $\mathrm{NH}_{3}$ & $0.71 \pm 0.05$ \\
\hline
\end{tabular}




\subsection{Implications for the origin of daughter species in $103 P$}

The results for $\mathrm{CN}$ and $\mathrm{NH}_{2}$ are consistent with an origin from dissociation from $\mathrm{HCN}$ and $\mathrm{NH}_{3}$, respectively, which is the current consensus on where these radicals originate. The results for $\mathrm{CH}$ suggest that a good portion of $\mathrm{CH}$ may have a source other than $\mathrm{CH}_{4}$. The variablity in the $\mathrm{CN} / \mathrm{H}_{2} \mathrm{O}$ and the $\mathrm{C}_{2} / \mathrm{CN}$ ratios (Figs. 7 and 8 , respectively, and Table 6 ) and the smaller amplitude of the variations in $Q_{\mathrm{C}_{2}}$ and $Q_{\mathrm{H}_{2} \mathrm{O}}$ compared to $Q_{\mathrm{CN}}$ (Figs. 5 and 6 and Table 5) suggest that $\mathrm{H}_{2} \mathrm{O}$ and $\mathrm{C}_{2}$ originate from icy or carbonaceous grains surrounding the nucleus, while the parent of $\mathrm{CN}$ is released directly from the nucleus. This is because the grains expand outward more slowly than the gas, meaning the grain population in the FOV will experience less rotational modulation than the number of gaseous molecules. Many observations have confirmed that a large majority of the $\mathrm{H}_{2} \mathrm{O}$ is released from icy grains (A'Hearn et al., 2011; Bonev et al., 2013; Knight and Schleicher, 2013).

The lesser rotational modulation of $\mathrm{C}_{2}$, combined with a lack of a sufficient amount of $\mathrm{C}_{2} \mathrm{H}_{2}$, suggests a significant fraction of the $\mathrm{C}_{2}$ production is coming from carbonaceous grains, also referred to as CHON particles. It is also possible that a hydrocarbon other than $\mathrm{C}_{2} \mathrm{H}_{2}$ is responsible and in turn this hydrocarbon is predominately present on the icy grains. However, any hydrocarbon other than $\mathrm{C}_{2} \mathrm{H}_{2}$ (and likely $\mathrm{C}_{2} \mathrm{H}_{2}$ as well) must release $\mathrm{C}_{2}$ through at least a 2-step decay process (Jackson, 1976; Cochran, 1985). Using spatial profiles of $C_{2}$ in $1 P /$ Halley, Combi and Fink (1997) showed that the observed spatial distribution could only be reproduced with a 2step decay process with improbably small ejection velocities for the daughter and granddaughter species. On the other hand, they determined that if $\mathrm{C}_{2}$ came predominately from a halo of $\mathrm{CHON}$ particles, the spatial distribution of $\mathrm{C}_{2}$ could be fit quite well. The lifetimes of $\mathrm{CHON}$ particles are comparable to the icy grains thought to be responsible for the $\mathrm{H}_{2} \mathrm{O}$ production in 103P, meaning it is likely any species originating from the $\mathrm{CHON}$ particles would have a similar rotational modulation to one produced from the icy grains, as we observe to be the case for $\mathrm{C}_{2}(\mathrm{CHON})$ and $\mathrm{H}_{2} \mathrm{O}$ (icy grains).

Another potential parent of $C_{2}$ is $C_{3}$. $C_{3}$ is a daughter species that has a bright emission band in the ARCES bandpass. However, accurate subtraction of the continuum under this feature is difficult because of the dense forest of lines present. Our method of continuum subtraction overestimates the continuum in the $\mathrm{C}_{3}$ emission region, resulting in an underestimation of the $C_{3}$ flux. For this reason we have excluded $C_{3}$ from the present analysis. However, measurements of $C_{3}$ in 103P from Knight and Schleicher (2013) suggest that $C_{3}$ may be abundant enough to account for the discrepency between the $\mathrm{C}_{2}$ and $\mathrm{C}_{2} \mathrm{H}_{2}$ production rates. However, $C_{3}$ is a daughter product, meaning that any $C_{2}$ released from $C_{3}$ photodissociation is released via a two-step process, which we argued in the previous paragraph is unlikely. If $C_{3}$ is released from photodissociation of a hydrocarbon such as $\mathrm{C}_{3} \mathrm{H}_{4}$, then $\mathrm{C}_{3}$ itself is likely a granddaughter or great-granddaughter species (Helbert et al., 2005; Weiler, 2012). Since all of these photodissociation events have long timescales of greater than $5 \times 10^{4} \mathrm{~s}$ (Helbert et al., 2005), this makes $C_{3}$ a very inefficient source of $C_{2}$ in the inner coma.

Therefore we consider our results another piece of evidence in favor of a $\mathrm{CHON}$ grain source for $\mathrm{C}_{2}$ in addition to photodissociation of hydrocarbons. Although some $\mathrm{C}_{2}$ does likely result from $\mathrm{C}_{2} \mathrm{H}_{2}$ photodissociation, at least for 103P a significant fraction of the $\mathrm{C}_{2}$ seems to originate from sublimating $\mathrm{CHON}$ particles or some other unknown source. Traditionally the $\mathrm{C}_{2} / \mathrm{CN}$ ratio has been considered a diagnostic for the abundunce of carbon-chain molecules. If indeed a majority of the $\mathrm{C}_{2}$ actually originates from $\mathrm{CHON}$ grains, the $C_{2}$ abundunce would then actually be a tracer for the abundunce of $\mathrm{CHON}$ particles present, not necessarily the carbon-chain molecule abundunce. This hypothesis also explains the possible tendency for comets beyond $2 \mathrm{AU}$ from the Sun to have lower $C_{2}$ abundunces than those at smaller heliocentric distance (Newburn and Spinrad, 1989), since beyond this distance CHON grains may not sublimate readily (Combi and Fink, 1997) (though other surveys, for example (A'Hearn et al., 1995), do not find evidence for such as a trend). An increase of the $\mathrm{C}_{2} / \mathrm{CN}$ ratio for comet C/2009 P1 Garradd inbound from 3 AU to $1.8 \mathrm{AU}$ is evident in optical spectra of this comet (McKay et al. in prep), which could be indicative of changing parentage for $C_{2}$ from exclusively photodissociating hydrocarbons to a combination of hydrocarbons and carbonaceous grains. More observations of $\mathrm{C}_{2}$ and $\mathrm{C}_{2} \mathrm{H}_{2}$ in comets are needed to fully understand the progeny of $\mathrm{C}_{2}$ in cometary comae.

As noted in the previous section, our $\mathrm{NH}_{2}$ production rates in September are lower than those of $\mathrm{NH}$ found by Knight and Schleicher (2013). Knight and Schleicher (2013) found that the spatial distribution of $\mathrm{NH}$ and $\mathrm{OH}$ were very similar, and suggested the $\mathrm{OH}$ and $\mathrm{NH}$ parents $\left(\mathrm{H}_{2} \mathrm{O}\right.$ and $\left.\mathrm{NH}_{3}\right)$ orignated from icy grains. However, if the changing $\mathrm{NH}_{2} / \mathrm{H}_{2} \mathrm{O}$ ratio inferred in Section 4.1 is real, this suggests that the $\mathrm{NH}_{2}$ parent $\left(\mathrm{NH}_{3}\right)$ comes from a source similar to the parent of $\mathrm{CN}(\mathrm{HCN})$, not $\mathrm{H}_{2} \mathrm{O}$. This combined with the production rates of $\mathrm{NH}$ found by Knight and Schleicher (2013) being systematically higher than our $\mathrm{NH}_{2}$ production rates may suggest that not all the $\mathrm{NH}$ in 103P's coma is a granddaughter product of $\mathrm{NH}_{3}$, but that some of it sublimates off of icy or carbonaceous grains. Either a spatial distribution of $\mathrm{NH}_{2}$ or a light curve for $\mathrm{NH}$ would be needed to test this possibility.

\subsection{Degree of compositional heterogeneity for the nucleus of $103 P$}

A'Hearn et al. (2011) observed that the $\mathrm{CO}_{2} / \mathrm{H}_{2} \mathrm{O}$ ratio for $103 \mathrm{P}$ changed by a factor of two over a nucleus rotation. Drahus et al. (2012) observed a similar trend for the $\mathrm{HCN} / \mathrm{CH}_{3} \mathrm{OH}$ ratio from sub-mm observations. Both A'Hearn et al. (2011) and Drahus et al. (2012) cite these changing mixing ratios as evidence for compositional heterogeneity in the nucleus. However, changing mixing ratios were not observed from ground-based IR observations (Dello Russo et al., 2011; Mumma et al., 2011; Kawakita et al., 2013). This may simply be due to the fact that the ground-based IR observations were "snapshot" observations, while those of A'Hearn et al. (2011) and Drahus et al. (2012) had longer temporal baselines that sampled the rotational variation better. Although they did not observe changing mixing ratios, ground-based IR observations did observe different spatial distributions for different species. $\mathrm{H}_{2} \mathrm{O}$ and $\mathrm{CH}_{3} \mathrm{OH}$ emission was extended in the antisolar direction, consistent with these molecules sublimating off icy grains, while the $\mathrm{HCN}, \mathrm{C}_{2} \mathrm{H}_{2}$, and $\mathrm{C}_{2} \mathrm{H}_{6}$ emission correlates with the $\mathrm{CN}$ jet seen in optical imaging (Knight and Schleicher, 2013). Therefore Mumma et al. (2011) and Kawakita et al. (2013) argue specifically from the different spatial distributions for different volatiles for small scale heterogeneity in that there are at least two different types of ice present in 103P, but that these ices are not necessarily distributed in a heterogeneous manner throughout the nucleus. Unfortunately $\mathrm{CO}_{2}$ cannot be observed from the ground, but it is interesting to note that $\mathrm{CH}_{3} \mathrm{OH}$ and $\mathrm{HCN}$, observed to have different amplitudes of rotational modulation in the sub- $\mathrm{mm}$, have different spatial distributions as observed in the IR. This suggests that species that have different spatial distributions should also have different amplitudes of rotational modulation in their production rates.

Our observations provide evidence from optical wavelengths that supports the interpretations of IR and sub-mm observations. We observed that the mixing ratios $\mathrm{CN} / \mathrm{H}_{2} \mathrm{O}$ and $\mathrm{C}_{2} / \mathrm{CN}$ (and possibly $\mathrm{NH}_{2} / \mathrm{H}_{2} \mathrm{O}$ ) are variable with rotational phase, just as A'Hearn et al. (2011) and Drahus et al. (2012) observed 
variability in the $\mathrm{CO}_{2} / \mathrm{H}_{2} \mathrm{O}$ and $\mathrm{HCN} / \mathrm{CH}_{3} \mathrm{OH}$ ratios, respectively. Since $\mathrm{HCN}$ and $\mathrm{H}_{2} \mathrm{O}$ exhibited different spatial distributions and we determined that $\mathrm{HCN}$ is likely the dominant source of $\mathrm{CN}$ in 103P's coma, it is not surprising that $\mathrm{CN}$ and $\mathrm{H}_{2} \mathrm{O}$ have different amplitudes of rotational modulation.

We suggest that changing mixing ratios with rotational phase can be explained by different volatiles having different sources, either directly from the nucleus or from the icy grain halo. This hypothesis also explains the different spatial distributions of different volatile species seen at both IR (Dello Russo et al., 2011; Mumma et al., 2011; Kawakita et al., 2013) and optical (Knight and Schleicher, 2013) wavelengths. Species outgassing directly from the nucleus exhibit spatial distributions similar to the $\mathrm{CN}$ jet, while species outgassing from the icy grain halo have spatial distributions that are always extended in the antisolar direction due to radiation pressure pushing the grains in that direction. Also, species outgassing directly from the nucleus will have stronger rotational modulation of their production rates than species originating from the icy grain halo due to the much shorter residence time of gas as compared to dust in a given FOV (see Section 4.3), resulting in changing mixing ratios of species that come from different sources. Therefore, a species that exhibits strong rotational modulation and has a spatial distribution correlated with the $\mathrm{CN}$ jet (e.g. $\mathrm{CN}, \mathrm{HCN}$ ) outgasses directly from the nucleus, while a species that exhibits weaker rotational modulation and has a spatial distribution extended in the antisolar direction (e.g. $\mathrm{H}_{2} \mathrm{O}$ ) originates predominately from the icy grain halo. This means that $\mathrm{H}_{2} \mathrm{O}, \mathrm{CH}_{3} \mathrm{OH}$, and $\mathrm{C}_{2}$ come from sources in the icy grain halo, while $\mathrm{CO}_{2}, \mathrm{CN}, \mathrm{HCN}, \mathrm{C}_{2} \mathrm{H}_{2}$, and $\mathrm{C}_{2} \mathrm{H}_{6}$ outgas directly from the nucleus. $\mathrm{CH}$ may originate in the icy grain halo and $\mathrm{NH}_{2}$ may outgas mostly from the nucleus, but more data is needed to test these conclusions.

The fact that there is a segregation of species between the icy grain halo and direct outgassing from the nucleus suggests that the composition of the ice sublimating in the icy grain halo is different than the ice that is sublimating directly off the nucleus. This supports the conclusions of Mumma et al. (2011) and Kawakita et al. (2013) pertaining to the heterogeneity of the ices in 103P.

\section{Conclusions}

We presented high spectral resolution optical spectra of Comet 103P/Hartley throughout the Fall 2010 apparition. We measured production rates for $\mathrm{CN}, \mathrm{C}_{2}, \mathrm{CH}, \mathrm{NH}_{2}$, and $\mathrm{H}_{2} \mathrm{O}$, and computed mixing ratios for these species compared to $\mathrm{H}_{2} \mathrm{O}$ and $\mathrm{CN}$. We observed significant rotational modulation in gas production rates (factors of 3-5 for all species). We also found evidence that the $\mathrm{CN} / \mathrm{H}_{2} \mathrm{O}, \mathrm{NH}_{2} /$ $\mathrm{H}_{2} \mathrm{O}$, and $\mathrm{C}_{2} / \mathrm{CN}$ ratios experience rotational modulation, although for $\mathrm{NH}_{2} / \mathrm{H}_{2} \mathrm{O}$ this required comparison of multiple data sets, which may introduce systematic errors due to different transitions observed and different observing geometry that are not considered in the formal error bars. There is also evidence that the $\mathrm{CH} / \mathrm{CN}$ ratio was higher pre-perihelion than post-perihelion.

We interpret the changing mixing ratios as a manifestation of the source of the gas, whether it sublimates directly from the nucleus or predominately from icy grains. In this picture, $C_{2}$ and $\mathrm{H}_{2} \mathrm{O}$ sublimate off $\mathrm{CHON}$ particles and icy grains, respectively. The parent molecules of $\mathrm{CN}$ and (possibly) $\mathrm{NH}_{2}$ sublimate directly from the nucleus. This suggests chemical heterogeneity in the composition of the ices present in the nucleus. By comparing our observations to those of parent molecules in the NIR, we determined that our observations are consistent with all $\mathrm{CN}$ and $\mathrm{NH}_{2}$ resulting from photodissociation of $\mathrm{HCN}$ and $\mathrm{NH}_{3}$, respectively. The $\mathrm{C}_{2}$ and $\mathrm{CH}$ abundances cannot be supplied by $\mathrm{C}_{2} \mathrm{H}_{2}$ and $\mathrm{CH}_{4}$ alone. Therefore another parent, whether a different hydrocarbon or CHON grains, is needed to account for the observed abundances.

\section{Acknowledgements}

We thank John Barentine, Jurek Krzesinski, Chris Churchill, Pey Lian Lim, Paul Strycker, and Doug Hoffman for developing and optimizing the ARCES IRAF reduction script used to reduce these data. We would also like to acknowledge the JPL Horizons System, which was used to generate ephemerides for nonsidereal tracking of the comets during the observations, and the SIMBAD database, which was used for selection of reference stars. We thank Hideyo Kawakita for providing us with the latest g-factors for $\mathrm{NH}_{2}$ and Dennis Bodewits for discussing results of the DIXI mission with us. This work is supported by the NASA GSRP Fellowship program through Grant No. NNX11AO03H. We appreciate the comments of two anonymous referees that improved the quality of this paper.

\section{References}

A'Hearn, M.F., Millis, R.L., Schleicher, D.G., Osip, D.J., Birch, P.V., 1995. The ensemble properties of comets: Results from narrowband photometry of 85 comets, 1976-1992. Icarus 118 (December), 223-270. http://dx.doi.org/10.1006/ icar.1995.1190.

A'Hearn, M.F. et al., 2011. EPOXI at Comet Hartley 2. Science 332 (June). http:// dx.doi.org/10.1126/science.1204054, 1396-.

Belton, M.J.S., Thomas, P., Li, J.-Y., Williams, J., Carcich, B., A'Hearn, M.F., McLaughlin, S., Farnham, T., McFadden, L., Lisse, C.M., Collins, S., Besse, S., Klaasen, K. Sunshine, J., Meech, K.J., Lindler, D., 2013. The complex spin state of 103P/ Hartley 2: Kinematics and orientation in space. Icarus 222 (February), 595-609. http://dx.doi.org/10.1016/j.icarus.2012.06.037.

Bhardwaj, A., Raghuram, S., 2012. A coupled chemistry-emission model for atomic oxygen green and red-doublet emissions in the Comet C/1996 B2 Hyakutake. Astrophys. J. 748 (March), 13. http://dx.doi.org/10.1088/0004-637X/748/1/13.

Bonev, B.P., Villanueva, G.L., Paganini, L., DiSanti, M.A., Gibb, E.L., Keane, J.V., Meech, K.J., Mumma, M.J., 2013. Evidence for two modes of water release in Comet 103P/Hartley 2: Distributions of column density, rotational temperature, and ortho-para ratio. Icarus 222 (February), 740-751. http://dx.doi.org/10.1016/ j.icarus.2012.07.034.

Cochran, A.L., 1985. C2 photolytic processes in cometary comae. Astrophys. J. 289 (February), 388-391. http://dx.doi.org/10.1086/162898.

Cochran, A.L., Cochran, W.D., 2002. A high spectral resolution atlas of Comet 122P/ de Vico. Icarus 157 (2), 297-308.

Combi, M., Harris, W., Smyth, W., 2004. Gas dynamics and kinetics in the cometary coma: Theory and observations. In: Comets II. University of Arizona Press, Tucson, AZ, USA, pp. 523-552.

Combi, M.R., Fink, U., 1997. A critical study of molecular photodissociation and CHON grain sources for cometary C 2. Astrophys. J. 484 (July), 879. http:// dx.doi.org/10.1086/304349.

Combi, M.R., Bertaux, J.-L., Quémerais, E., Ferron, S., Mäkinen, J.T.T., 2011. Water Production by Comet 103P/Hartley 2 Observed with the SWAN Instrument on the SOHO Spacecraft. Astrophys. J. 734 (June), L6. http://dx.doi.org/10.1088/ 2041-8205/734/1/L6.

Dello Russo, N. et al., 2009. The parent volatile composition of $6 \mathrm{p} / \mathrm{d}$ ' arrest and a chemical comparison of Jupiter-family comets measured at infrared wavelengths. Astrophys. J. 703 (September), 187. http://dx.doi.org/10.1088/ 0004-637X/703/1/187.

Dello Russo, N., Vervack Jr., R.J., Kawakita, H., Kobayashi, H., Keck Observatory, W.M., 2010. Comet 103P/Hartley. IAU Circ. 91 (September), 1.

Dello Russo, N. et al., 2011. The volatile composition and activity of Comet 103P/ Hartley 2 during the EPOXI closest approach. Astrophys. J. 734 (June). http:/ dx.doi.org/10.1088/2041-8205/734/1/L8, L8+.

Drahus, M., Jewitt, D., Guilbert-Lepoutre, A., Waniak, W., Sievers, A., 2012. The sources of $\mathrm{HCN}$ and $\mathrm{CH}_{3} \mathrm{OH}$ and the rotational temperature in Comet 103P/ Hartley 2 from time-resolved millimeter spectroscopy. Astrophys. J. 756 (September), 80. http://dx.doi.org/10.1088/0004-637X/756/1/80.

Farnham, T.L., Schleicher, D.G., A'Hearn, M.F., 2000. The HB narrowband comet filters: Standard stars and calibrations. Icarus 147 (September), 180-204. http://dx.doi.org/10.1006/icar.2000.6420.

Feaga, L.M., A'Hearn, M.F., Sunshine, J.M., Groussin, O., Farnham, T.L., 2007 Asymmetries in the distribution of $\mathrm{H}_{2} \mathrm{O}$ and $\mathrm{CO}_{2}$ in the inner coma of Comet 9P/Tempel 1 as observed by Deep Impact. Icarus 190 (2), 345-356.

Festou, M.C., 1981. The density distribution of neutral compounds in cometary atmospheres I - models and equations. Astron. Astrophys. 95 (1), 69-79.

Filippenko, A.V., 1982. The importance of atmospheric differential refraction in spectrophotometry. Publ. Astron. Soc. Pacific 94 (August), 715-721. http:// dx.doi.org/10.1086/131052.

Haser, L., 1957. Distribution d'intensite dans la tete d'une comete. Bull. Acad. R. Sci. Liege 43, 740-750. 
Helbert, J., Rauer, H., Boice, D.C., Huebner, W.F., 2005. The chemistry of $C_{2}$ and $C_{3}$ in the coma of Comet C/1995 O1 (Hale-Bopp) at heliocentric distances $r_{h} \geqslant 2.9 \mathrm{AU}$. Astron. Astrophys. 442 (November), 1107-1120. http:// dx.doi.org/10.1051/0004-6361:20041571.

Huebner, W.F., Keady, J.J., Lyon, S.P., 1992. Solar photo rates for planetary atmospheres and atmospheric pollutants. Astrophys. Space Sci. 195 (1), 1 289, 291-294.

Jackson, W.M., 1976. The photochemical formation of cometary radicals. J. Photochem. 5 (2), 107-118.

Kawakita, H., Watanabe, J.-i., 2002. Revised fluorescence efficiencies of cometary $\mathrm{NH}_{2}$ : Ammonia abundance in comets. Astrophys. J. 572 (June), L177-L180. http://dx.doi.org/10.1086/341746.

Kawakita, H., Kobayashi, H., Dello Russo, N., Vervack, R.J., Hashimoto, M., Weaver, H.A., Lisse, C.M., Cochran, A.L., Harris, W.M., Bockelée-Morvan, D. Biver, N., Crovisier, J., McKay, A.J., 2013. Parent volatiles in Comet 103P/ Hartley 2 observed by Keck II with NIRSPEC during the 2010 apparition. Icarus 222 (February), 723-733. http://dx.doi.org/10.1016/ j.icarus.2012.08.006.

Knight, M.M., Schleicher, D.G., 2011. CN morphology studies of Comet 103P/Hartley 2. Astron. J. 141 (June), 183. http://dx.doi.org/10.1088/0004-6256/141/6/183.

Knight, M.M., Schleicher, D.G., 2013. The highly unusual outgassing of Comet 103P/ Hartley 2 from narrowband photometry and imaging of the coma. Icarus 222 (February), 691-706. http://dx.doi.org/10.1016/j.icarus.2012.06.004.

Kobayashi, H. et al., 2010. High-dispersion infrared spectroscopic observations of Comet 8P/Tuttle with VLT/CRIRES. Astron. Astrophys. 509 (January), A80. http:// dx.doi.org/10.1051/0004-6361/200912477.

Magee-Sauer, K., Scherb, F., Roesler, F.L., Harlander, J., 1990. Comet Halley O $\left({ }^{1}\right.$ D) and $\mathrm{H}_{2} \mathrm{O}$ production rates. Icarus 84 (March), 154-165. http://dx.doi.org/10.1016/ 0019-1035(90)90163-4.

Markwardt, C.B., 2009. Non-linear least-squares fitting in IDL with MPFIT. In: Bohlender, D.A., Durand, D., Dowler, P. (Eds.), Astronomical Data Analysis Software and Systems XVIII, vol. 411. ASP Conf. Ser., San Francisco, CA, September, ASP, pp. 251-255.

McKay, A.J., Chanover, N.J., Morgenthaler, J.P., Cochran, A.L., Harris, W.M., Russo N.D., 2012. Forbidden oxygen lines in Comets C/2006 W3 Christensen and C/ 2007 Q3 Siding Spring at large heliocentric distance: Implications for the sublimation of volatile ices. Icarus 220 (July), 277-285. http://dx.doi.org/ 10.1016/j.icarus.2012.04.030.

McKay, A.J., Chanover, N.J., Morgenthaler, J.P., Cochran, A.L., Harris, W.M., Russo, N.D., 2013. Observations of the forbidden oxygen lines in DIXI target Comet 103P/Hartley. Icarus 222 (February), 684-690. http://dx.doi.org/10.1016/ j.icarus.2012.06.020.

Meech, K.J. et al., 2011. EPOXI: Comet 103P/Hartley 2 observations from a worldwide campaign. Astrophys. J. 734 (June). http://dx.doi.org/10.1088/ 2041-8205/734/1/L1, L1+.
Morgenthaler, J.P., Harris, W.M., Combi, M.R., 2007. Large aperture O I $6300 \AA$ observations of Comet Hyakutake: Implications for the Photochemistry of $\mathrm{OH}$ and OI production in Comet Hale-Bopp. Astrophys. J. 657 (March), 1162-1171. http://dx.doi.org/10.1086/511062.

Morgenthaler, J.P. et al., 2001. Large aperture [OI] $6300 \AA$ photometry of comet Hale-Bopp: Implications for the photochemistry of OH. Astrophys. J. 563 (1), 451-461.

Mumma, M.J. et al., 2011. Temporal and spatial aspects of gas release during the 2010 apparition of Comet 103P/Hartley 2. Astrophys. J. 734 (June), L7. http:// dx.doi.org/10.1088/2041-8205/734/1/L7.

Newburn, R.L., Spinrad, H., 1989. Spectrophotometry of 25 comets - Post-Halley updates for 17 comets plus new observations for eight additional comets. Astron. J. 97 (February), 552-569. http://dx.doi.org/10.1086/115005.

Samarasinha, N.H., Mueller, B.E.A., A'Hearn, M.F., Farnham, T.L., Gersch, A., 2011. Rotation of Comet 103P/Hartley 2 from structures in the coma. Astrophys. J. 734 (June), L3. http://dx.doi.org/10.1088/2041-8205/734/1/L3.

Schleicher, D.G., 2010. The fluorescence efficiencies of the $\mathrm{CN}$ violet bands in comets. Astron. J. 140 (October), 973-984. http://dx.doi.org/10.1088/00046256/140/4/973.

Schultz, D., Li, G.S.H., Scherb, F., Roesler, F.L., 1992. Comet Austin (1989c1) O( $\left.{ }^{1} D\right)$ and $\mathrm{H}_{2} \mathrm{O}$ production rates. Icarus 96 (April), 190-197. http://dx.doi.org/ 10.1016/0019-1035(92)90072-F.

Shinnaka, Y., Kawakita, H., Kobayashi, H., Naka, C., Arai, A., Arasaki, T., Kitao, E., Taguchi, G., Ikeda, Y., 2013. Optical low-dispersion spectroscopic observations of Comet 103P/Hartley 2 at Koyama Astronomical Observatory during the EPOXI flyby. Icarus 222 (February), 734-739. http://dx.doi.org/10.1016/ j.icarus.2012.08.001.

Singh, P.D., Dalgarno, A., 1987. Photodissociation lifetimes of $\mathrm{CH}$ and $\mathrm{CD}$ radicals in comets. In: Rolfe, E.J., Battrick, B. (Eds.), Diversity and Similarity of Comets, vol. 278. ESA Special Publication, pp. 177-179.

Spinrad, H., 1982. Observations of the red auroral oxygen lines in nine comets. Publ. Astron. Soc. Pacific 94 (December), 1008-1016. http://dx.doi.org/10.1086/ 131101.

Swings, P., 1941. Complex structure of cometary bands tentatively ascribed to the contour of the solar spectrum. Lick Observ. Bull. 19, 131-136.

Tseng, W.-L., Bockelée-Morvan, D., Crovisier, J., Colom, P., Ip, W.-H., 2007. Cometary water expansion velocity from $\mathrm{OH}$ line shapes. Astron. Astrophys. 467 (May), 729-735. http://dx.doi.org/10.1051/0004-6361:20066666.

Villanueva, G.L., Mumma, M.J., Disanti, M.A., Bonev, B.P., Gibb, E.L., Magee-Sauer, K., Blake, G.A., Slayk, C., 2011. The molecular composition of Comet C/2007 W1 (Boattini): Evidence of a peculiar outgassing and a rich chemistry. Icarus 216 (November), 227-240. http://dx.doi.org/10.1016/j.icarus.2011.08.024.

Weiler, M., 2012. The chemistry of $C_{3}$ and $C_{2}$ in cometary comae. I. Current models revisited. Astron. Astrophys. 538 (February), A149. http://dx.doi.org/10.1051/ 0004-6361/201117480. 